\title{
Adaptable Bandwidth for Harmonic Step-Frequency Radar
}

\author{
Anthony F. Martone, ${ }^{1}$ Kyle A. Gallagher, ${ }^{2}$ Kelly D. Sherbondy, ${ }^{1}$ Kenneth I. Ranney, \\ Traian V. Dogaru, ${ }^{1}$ Gregory J. Mazzaro, ${ }^{3}$ and Ram M. Narayanan ${ }^{2}$ \\ ${ }^{1}$ U.S. Army Research Laboratory, 2800 Powder Mill Road, Adelphi, MD 20783, USA \\ ${ }^{2}$ Department of Electrical Engineering, The Pennsylvania State University, University Park, PA 16802, USA \\ ${ }^{3}$ Department of Electrical and Computer Engineering, The Citadel, Charleston, SC 29409, USA
}

Correspondence should be addressed to Anthony F. Martone; anthony.f.martone.civ@mail.mil

Received 10 April 2015; Accepted 29 June 2015

Academic Editor: Ahmed A. Kishk

Copyright (C) 2015 Anthony F. Martone et al. This is an open access article distributed under the Creative Commons Attribution License, which permits unrestricted use, distribution, and reproduction in any medium, provided the original work is properly cited.

A spectrum sensing technique is described which is used to enhance the performance of harmonic step-frequency radar in the presence of harmful radio frequency (RF) interference (RFI). This technique passively monitors the RF spectrum for subbands of high signal-to-interference-plus-noise ratio (SINR) within a constrained bandwidth of interest. An optimal subband is selected for the harmonic radar that maximizes SINR and minimizes the range resolution cell size, two conflicting objectives. The approach is tested using an experimental setup that injects high power RFI into a harmonic step-frequency radar, which significantly degrades radar performance. It is shown that the proposed spectrum sensing technique significantly improves the SINR and the peak-toaverage sidelobe power level of the harmonic radar at the sacrifice of range resolution.

\section{Introduction}

The ever-growing wireless communications industry poses several radio frequency (RF) challenges for radar systems [1, 2]. One such challenge is RF interference (RFI) caused by RF sources operating in band and out of band to the radar [3]. It has been shown that RFI significantly degrades performance in multiple radar types including Next-Generation Weather Radars (NEXRADs) [4], air traffic control radars [5], through-the-wall radar [6], microwave radiometers [7], and harmonic radar [8]. Future radars must therefore be capable of detecting and mitigating RFI in order to maximize radar performance.

Another challenge is that the radar can itself become a source of interference, a known problem for ultrawideband radars interfering with external RF sources $[9,10]$. If no cooperative policy is in place, then radar transmissions may directly (by operating in the same band) or indirectly (by outof-band emissions) interfere with RF systems operating in the vicinity. As such, future radars must be capable of automatically identifying and cooperating with other RF systems. A final challenge for radar is adherence to the potential new
FCC regulations specified by the National Broadcast Plan, a plan to ensure "every American has access to broadband capability [11]." One aspect of these regulations is to free $500 \mathrm{MHz}$ of federal and nonfederal spectra for mobile and fixed wireless broadband usage [12]. It remains unclear as to how these regulations will be implemented and what frequency bands will be affected. However, any radar currently operating in the affected frequency band will be rendered useless. Clearly radars need a way to coexist in the EM environment by (1) detecting and mitigating interference from RF sources; (2) identifying and/or cooperating with RF systems in order to avoid causing interference; (3) modifying the radars' bandwidth to comply with domestic and international regulations.

The radar type of interest in this development is harmonic, step-frequency radar for RF device identification [13]. Harmonic radars receive and process harmonically generated radar returns that occur at multiples of the transmit frequency resulting from nonlinear scattering by targets of interest [14]. It has the advantage over traditional linear radar of separating natural clutter from the induced nonlinear response. This separation is made possible by the properties 
inherent to the nonlinear components in the target of interest; semiconductors and metal-to-metal junctions are known sources of nonlinearity $[15,16]$. Hence, processing nonlinear target responses offers an alternative way to detect and locate a target of interest. For example, in harmonic radar RF identification (RFID) applications, such as insect tracking [17], a nonlinear radar tag (typically a diode connected to a transducer) is attached to the target of interest; this tag is specially designed to rebroadcast a harmonic response for detection. Ranging of the nonlinear target requires the harmonic radars receiver bandwidth to be a harmonic multiple of the transmit bandwidth. For example, if the harmonic radar transmits between 800 and $1000 \mathrm{MHz}$, then the receiver bandwidth is 1600-2000 MHz. Only objects containing nonlinearities can be detected in the receiver bandwidth and all other responses (e.g., clutter) remain in the linear band.

RFI is particularly disruptive to harmonic radar since the signal-to-noise ratio of the second order nonlinearity decreases at a rate of $1 / R^{6}$ in free space for two-way propagation, not $1 / R^{4}$ as is the case with traditional linear radar [18]. Therefore, if RFI occupies frequency bins used to receive the low-powered nonlinear response, then the signal-tointerference-plus-noise ratio (SINR) decreases; reception at these frequencies must be avoided. In addition, the RF components of the harmonic radar are inherently highly sensitive [19]. The harmonic radar must therefore avoid interference that could (1) saturate the RF components and (2) drive the $\mathrm{RF}$ components into their nonlinear operating region causing self-induced harmonics.

Potential solutions for mitigating RFI in radar include [6] (1) random frequency selection [20], (2) frequency notching methods [21], (3) subspace techniques [22], and (4) RFI reconstruction and subtraction [23]. As discussed in [6], the effectiveness of these solutions requires an estimate of the RFI parameters, a challenging task. Furthermore, these solutions do not address the second (i.e., radar as the RFI source) and third (i.e., modifiable radar bandwidth used to comply with government regulations) radar challenges discussed in the opening paragraph. To address these two challenges, joint radar and radio spectrum sharing techniques have been proposed for coexistence within a fixed frequency band of interest $[24,25]$. One such technique considers a bandwidth sharing approach for radar and communications [26]. This technique allows the radar to utilize as much bandwidth as needed within a fixed frequency band of interest (the radar is given priority over the communications system).

A potential solution that addresses all of the above radar challenges is the spectrum sensing, multiobjective optimization (SS-MO) technique originally introduced by the authors in [27]. This technique passively monitors the operating band of the radar for RFI while the radar remains inactive. The technique then identifies a frequency subband (within operating band of the radar) with minimal RFI. The SS-MO technique meets optimization criteria that require a maximum SINR and a minimum range resolution cell size, two conflicting objectives. A minimized range resolution cell size is beneficial to radar since it is used to maximize the signalto-clutter ratio (SCR) in distributed clutter scenarios and enhance features for target classification [28]. The radar next modifies its waveform bandwidth to operate in the frequency subband selected by the proposed technique. Any waveform that supports a variable bandwidth can be utilized by this technique. As discussed in [27], this proposed technique was used to analyze multiple frequency spectra of different spectral occupancy collected by a spectrum monitor system. The model used in that analysis assumed constant radar parameters; no radar data were collected or processed for experimentation. Analysis of the frequency spectra indicated that the proposed technique successfully identifies frequency subbands of high SINR while maintaining range resolution requirements. We concluded that further investigation was required in order to understand how the proposed technique affects radar performance.

In this paper, the SS-MO technique is extended to harmonic step-frequency radar. A radar performance trade-off study is conducted to determine the effectiveness of the technique on radar data. The radar performance metrics consist of SINR, peak-to-average sidelobe ratio, and range resolution cell size. Section 2 provides the formulation of the harmonic step-frequency waveform model. Section 3 describes the proposed SS-MO technique and how this technique is applied to harmonic step-frequency radar. Section 4 describes the closed-loop, harmonic step-frequency radar data acquisition system and experiments. This system measures the harmonic response from a nonlinear circuit element (i.e., a passive amplifier) in the presence of RFI. The RFI consists of deterministic narrowband and wideband signals. Section 5 discusses the advantages of the proposed approach and provides a conclusion to the paper.

\section{Harmonic Step-Frequency Waveform Formulation}

A block diagram of the harmonic step-frequency system model is shown in Figure 1. This model is adapted from [29] for distortion prediction of nonlinear systems. Note that this model does not consider over-the-air waveform propagation and the effects of signal attenuation due to range but is appropriately matched to the wired experiments discussed in Section 4 . The transmit signal of the step-frequency radar is defined as

$$
S_{1}(n, t)=\Lambda_{1} e^{2 \pi f(n) t+\phi},
$$

where $\Lambda_{1}$ is the amplitude of the signal, $\phi$ is the phase, $0 \leq$ $t \leq \tau$ is time, $\tau$ is pulse width, $f(n)=f_{o}+(n-1) F_{\Delta}$ is the frequency for the $n$th pulse, $f_{o}$ is the carrier frequency of the first pulse, $F_{\Delta}$ is the frequency step size, $n$ is an integer defined in the range $1 \leq n \leq N$, and $N$ is the number of pulses (or steps) [30]. The effective bandwidth of (1) is defined as $B=$ $f(N)-f(1)$.

The transmit signal is next input to a linear, time invariant (LTI) system as shown in Figure 1. In the experiments that follow (Section 4), this LTI system is used to produce a highly linear transmit waveform in order to prevent self-generated harmonics. The self-generated harmonics are indistinguishable from the harmonics generated by the target and must 


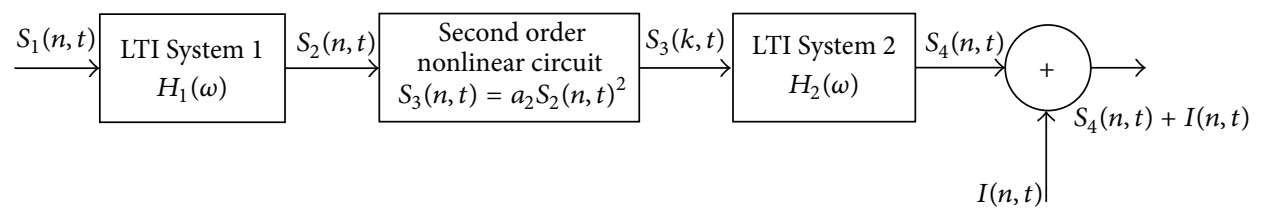

FIGURE 1: Block diagram of the harmonic step-frequency radar model with added deterministic interference. This model is adapted from [29] for distortion prediction of nonlinear systems.

therefore be attenuated. The response from LTI System 1 is defined as [29]

$$
\begin{aligned}
S_{2}(n, t) & =\int_{-\infty}^{\infty} h_{1}(\xi) S_{1}(n, t-\xi) d \xi \\
& =S_{1}(n, t) \int_{-\infty}^{\infty} h_{1}(\xi) e^{-[j 2 \pi f(n)] \xi} d \xi \\
& =S_{1}(n, t) H_{1}(j 2 \pi f(n)) \\
& =S_{1}(n, t)\left|H_{1}(j 2 \pi f(n))\right| e^{j \phi_{H 1}[j 2 \pi f(n)]} \\
& =\Lambda_{2} e^{j\left[2 \pi f(n) t+\phi_{2}\right]},
\end{aligned}
$$

where $h_{1}(t)$ is the system impulse response, $H_{1}(\omega)$ is the system transfer function, $\left|H_{1}(\omega)\right|$ is the magnitude of $H_{1}(\omega)$, $\phi_{H 1}(\omega)$ is the phase of $H_{1}(\omega), \Lambda_{2}(n)=\Lambda_{1}\left|H_{1}(j 2 \pi f(n))\right|$, and $\phi_{2}(n)=\phi+\phi_{H 1}(j 2 \pi f(n))$.

A model commonly used for a nonlinear circuit element is the memoryless, infinite power series [8]: $S_{3}(n, t)=$ $\sum_{i=1}^{\infty} a_{i}\left[S_{2}(n, t)\right]^{i}$, where $a_{i}$ is the amplitude coefficient of the $i$ th harmonic and a complex number. The values of the amplitude coefficients are dependent on the properties of the nonlinear target. The infinite power series model is reduced to the second order term for harmonic radar and the output to the nonlinearity becomes

$$
S_{3}(n, t)=a_{2}\left[S_{2}(n, t)\right]^{2}=a_{2} \Lambda_{2}(n)^{2} e^{2 j\left[2 \pi f(n) t+\phi_{2}(n)\right]} .
$$

Observe from (3) that the frequency and phase of the input signal double due to the second order nonlinearity. This observation is discussed in more detail at the end of this section.

The response from the nonlinearity is next input into LTI System 2 representing the receiver channel of the radar receiver. Following the same development in (2), the response of LTI System 2 is

$$
\begin{aligned}
& S_{4}(n, t)=S_{3}(n, t) H_{2}(j 4 \pi f(n)) \\
& \quad=\Lambda_{4}(n) e^{j\left(2 \pi[2 f(n)] t+2 \phi+2 \phi_{H 1}[j 2 \pi f(n)]+\phi_{H 2}[j 4 \pi f(n)]\right)},
\end{aligned}
$$

where $H_{2}(\omega)$ is the system transfer function,

$$
\begin{aligned}
\Lambda_{4}(n) & =a_{2} \Lambda_{2}(n)^{2}\left|H_{2}(j 4 \pi f(n))\right| \\
& =a_{2} \Lambda_{1}^{2}\left|H_{1}(j 2 \pi f(n))\right|^{2}\left|H_{2}(j 4 \pi f(n))\right|,
\end{aligned}
$$

$\left|H_{2}(j 4 \pi f(n))\right|$ is the magnitude of $H_{2}(\omega)$, and $\phi_{H_{2}}[j 4 \pi f(n)]$ is the phase of $H_{2}(\omega)$. RFI power is next added to (4). For this development the RFI is considered deterministic and only affects $S_{4}(n, t)$; that is, the RFI does not drive the nonlinear circuit model. The RFI is defined as

$$
I(n, t)=\Lambda_{\mathrm{RFI}}(n) e^{2 \pi f_{\mathrm{RFI}}(n) t+\phi_{\mathrm{RFI}}(n)},
$$

where $A_{\mathrm{RFI}}(n)$ is the RFI amplitude, $f_{\mathrm{RFI}}(n)$ is the RFI frequency, and $\phi_{\mathrm{RFI}}(n)$ is the RFI phase. It will be shown in the following section that the RFI power is empirically estimated using passive sensing for radar.

Examination of (4) indicates that the frequencies of the received signals are $\bar{f}(n)=2 f(n)$, twice that of the linear transmit frequencies defined in $f(n)$. Note that the frequency step size becomes $\bar{F}_{\Delta}=2 F_{\Delta}$. Furthermore, the bandwidth in (9) is defined as

$$
\bar{B}=2 B
$$

and is twice the bandwidth of the transmit step-frequency waveform defined by (1). As a result, the range resolution cell size is

$$
R_{\text {res }}=\frac{c}{2 \bar{B}}=\frac{c}{4 B}
$$

and is half the resolution cell size of (1).

\section{Spectrum Sensing, Multiobjective Optimization Technique}

In this section, the SS-MO technique introduced in [27] is adapted for harmonic step-frequency radar and explained in detail throughout this section. A block diagram of the proposed SS-MO technique is illustrated in Figure 2. As is shown, spectrum sensing is used to monitor the received frequency band of the harmonic radar and form an empirical estimate of the interference-plus-noise power. The empirical estimate of the interference-plus-noise power is based on energy detection [31]. Energy detection is used since it offers a means to detect a wide variety of interferences from multiple RF sources. This estimate is next used in conjunction with an estimate of the received power to form the SINR objective function. A range resolution objective function is determined a priori based on the radar requirements. Both the SINR and range resolution objective functions are fed into a linear weighting function for multiobjective optimization. A subband (within the total bandwidth) and its center frequency are identified by the linear weighting function and 


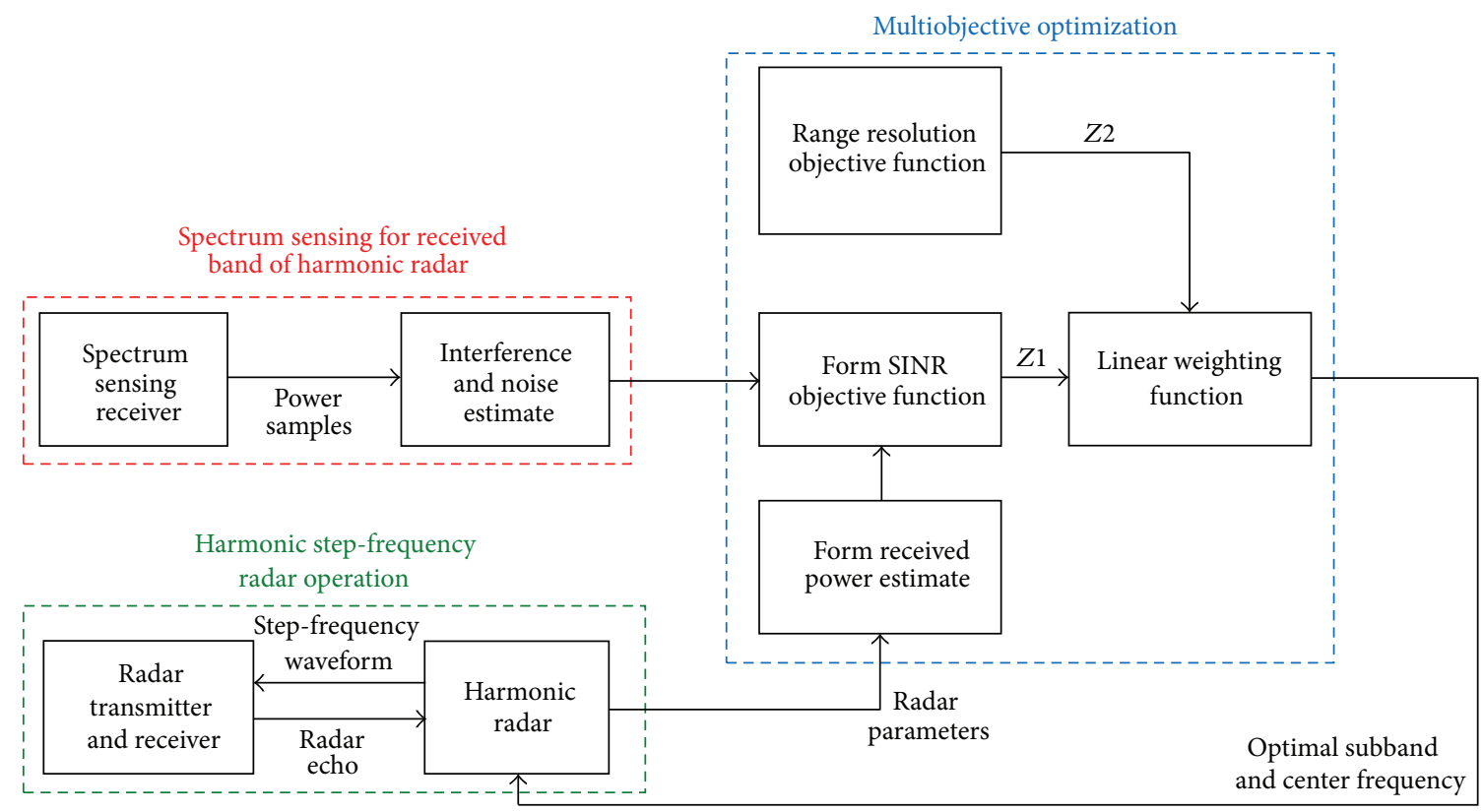

FIGURE 2: Proposed SS-MO technique for harmonic step-frequency radar. This technique requires an empirical measure of the RF spectrum. Multiobjective optimization is used to find a subband within the operational frequency band of the radar.

fed back to the radar system where the appropriate waveform is synthesized.

The timing of the proposed SS-MO technique is defined as

$$
T=T_{s}+T_{o}+T_{r}
$$

where $T$ is the pulse repetition interval (PRI), $T_{s}$ is the spectrum sensing processing time, $T_{o}$ is the multiobjective optimization processing time, and $T_{r}$ is the radar operation time. It is required that spectrum sensing and multiobjective optimization are implemented before radar operation so that the radar has an up-to-date measure of the RFI. The computational complexity of the proposed SS-MO technique should be minimal since it impacts the PRI and radar performance; for example, a high complexity may lead to a reduced number of pulses on target and therefore decrease the radar SINR.

The spectrum sensing receiver is used to passively monitor the fixed frequency band $B$, that is, the received band of the harmonic step-frequency radar. An analog frontend is used to receive, process, and digitize the observed interference and noise to generate a sequence of time domain samples. The fast Fourier transform (FFT) then processes these samples to generate frequency domain samples $X=$ $\left\{X_{1}, \ldots, X_{n}\right\}$ corresponding to the harmonic frequencies in $\bar{f}(n)$. The frequency resolution is defined as $\bar{F}_{\Delta}$, the frequency step size. Power estimates of the samples in $X$ are next defined as $\theta=\left\{\theta_{1}, \ldots, \theta_{n}\right\}$, where the $k$ th estimate is defined as $\theta_{k}=\left|X_{k}\right|^{2}$. Note that the samples in $\theta$ constitute an empirical estimate of the power spectrum of $I(n, t)$ defined in (6).

Interference-plus-noise estimates are next determined for all subband combinations within the received band of the harmonic step-frequency radar; it is again noted that this estimate is empirically calculated based on an observation of the RF spectrum. A variation of Pascal's triangle [32] is used to determine the total energy in all possible subbands. An example of this variation is shown in Figure 3 for $N=5$ power estimates $\left\{\theta_{1}, \ldots, \theta_{5}\right\}$. Each level in this triangle corresponds to a particular subband size. Each element in a given level corresponds to a particular center frequency. Level 1 elements at the top of the triangle contain the power estimates. As is indicated by the black arrows in Figure 3, Level 1 elements are summed together to form Level 2 elements. Level 3 elements require a sum between the elements of Level 2 and Level 1 as indicated by the solid and dashed blue lines (resp.). Level 4 elements require a sum between Level 3 and Level 1 elements as indicated by the solid and dashed green lines. The same procedure is used to form the elements of Level 5 (red lines).

The example of Figure 3 can be expanded from 5 to $N$ levels using the following recursive formula:

$$
\begin{aligned}
& \Gamma_{i, j} \\
& = \begin{cases}\theta_{j}, & i=1 ; j=1, \ldots, N, \\
\Gamma_{1, j}+\Gamma_{1, j+1}, & i=2 ; j=1, \ldots, N-1, \\
\Gamma_{i-1, j}+\Gamma_{1, i+j-1}, & i=3, \ldots, N ; j=1, \ldots, N-i+1,\end{cases}
\end{aligned}
$$

where $i \leq N$ represents the level number and $j \leq N$ represents the element location for a given Level $i$. The bandwidth for any level, that is, the subband size, is determined as

$$
\beta_{i, j}=i \bar{F}_{\Delta}
$$




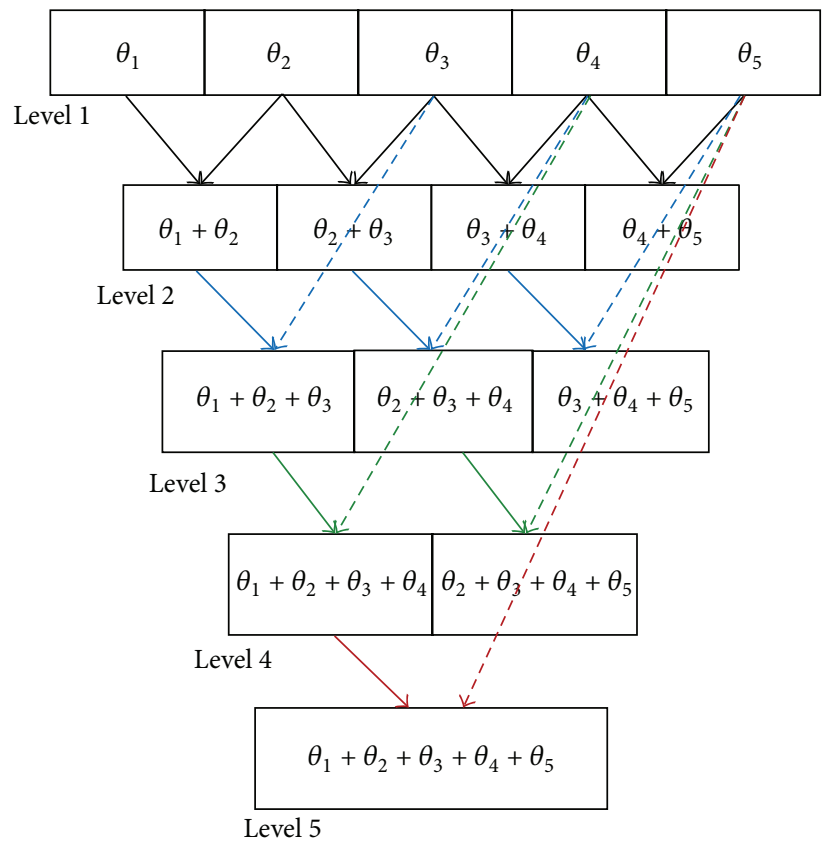

FIGURE 3: Example triangle structure used to determine the total power in all subband combinations. This structure is a variation of Pascal's triangle.

for $i=\{1, \ldots, N\}, j=\{1, \ldots, N-i+1\}$, and $\beta_{i, j} \subset \bar{B}$. The center frequency of any element for a given level is defined as

$$
f_{c}=\frac{[\bar{f}(j)+\bar{f}(j+i-1)]}{2},
$$

for $i=\{1, \ldots, N\}, j=\{1, \ldots, N-i+1\}$. The computational complexity of $(10)$ requires $\left(N^{2}-N\right) / 2$ summations.

In this development it is necessary to consider the average power within each subband and not the total power as calculated by (10). Recall that the receiver thermal noise is constant based on the frequency resolution. The average power is therefore needed so that thermal noise does not accumulate as $\beta_{i, j}$ grows larger. The average interferenceplus-noise power is defined as

$$
\widehat{\Gamma}_{i, j}=\frac{\Gamma_{i, j}}{i}
$$

where the number of elements in (13) is determined as

$$
\dot{N}=\sum_{n=1}^{N}(N-n+1)=\frac{\left(N^{2}+N\right)}{2} \text {, }
$$

which represents the total number of elements (i.e., subbands) available. The computational complexity of (13) requires $\dot{N}-N$ divisions.

The interference and noise power estimate in (13) is next used in conjunction with the signal power estimated from (5), that is, $\left|\Lambda_{4}(n)\right|^{2}$, to derive the SINR objective function. For this development, the magnitude of the system transfer functions defined in (5) is considered frequency independent such that $\left|H_{1}\right|=\left|H_{1}(j 2 \pi f(n))\right|,\left|H_{2}\right|=\left|H_{2}(j 4 \pi f(n))\right|$, and $\Lambda_{4}(n)=\Lambda_{4}$. The SINR objective function is defined as

$$
Z 1_{i, j}=\frac{\operatorname{Pr}_{i, j}}{\widehat{\Gamma}_{i, j}}
$$

where $\operatorname{Pr}_{i, j}=\left|\Lambda_{4}\right|^{2} /(2 \Omega)$ for $i=\{1, \ldots, N\}, j=\{1, \ldots, N-i+$ $1\}$, and $\Omega$ is the load impedance. The number of elements in $\operatorname{Pr}_{i, j}$ is $\dot{N}$, the same as for $\widehat{\Gamma}_{i, j}$. The computational complexity needed to form (15) requires $\dot{N}$ divisions. The minimum value of (15), $Z 1_{\text {min }}$, is predefined and corresponds to the minimum SINR needed by the radar for target detection.

The range resolution objective function is defined as the reciprocal of the range resolution using the bandwidth $\beta_{i, j}$ :

$$
Z 2_{i, j}=\frac{2 \beta_{i, j}}{c} .
$$

One goal of the optimizer is to maximize (16). The number of elements in $Z 2_{i, j}$ is $\dot{N}$, the same as for $Z 1_{i, j}$. Note that (16) is computed a priori and is retrieved from memory as needed (no computations needed). The minimum value of (16), $Z 2_{\text {min }}$, is predefined and corresponds to the worst range resolution available for the radar. The maximum value of (16) occurs when the subband equals the total available bandwidth, $\beta_{i, j}=\bar{B}$, and is defined as $Z 2_{\max }=2 \bar{B} / c$. This maximum value corresponds to the best range resolution available to the radar.

Notice that bandwidth is a parameter in both (15) and (16), which sets up a fundamental conflict. In order to minimize the range resolution cell size, we require that $\beta_{i, j}$ is as large as possible. This implies, however, that we increase 


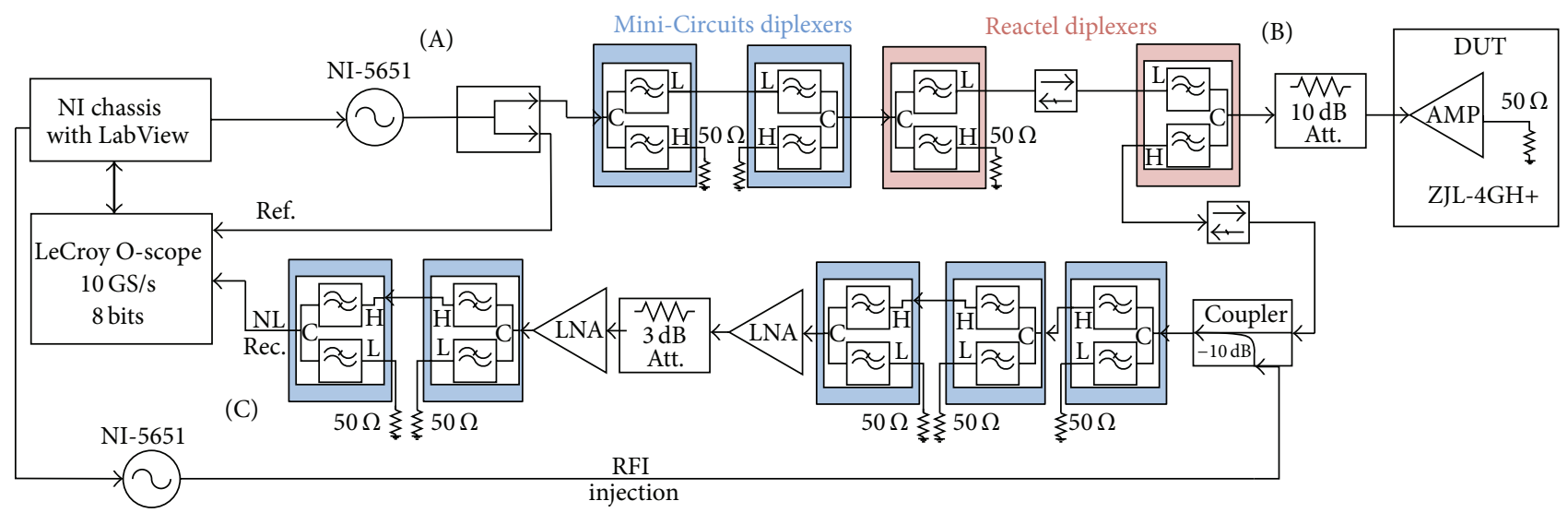

FIGURE 4: Block diagram of the harmonic step-frequency radar measurement system. This system is specially designed to reject unwanted self-generated interference and produce highly linearized transmit waveforms.

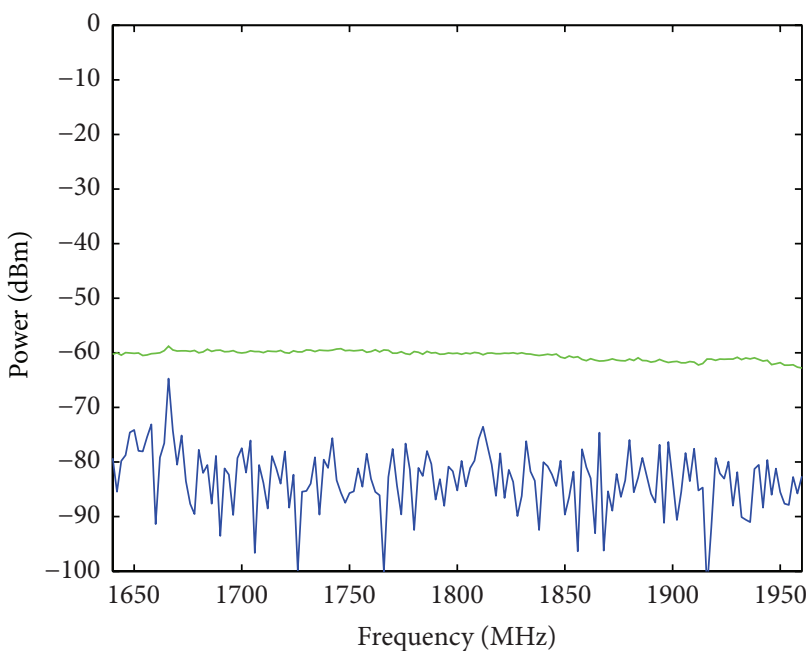

(a) The green plot is the power spectrum of the measured received signals when the DUT is present. The blue plot is the power spectrum of the measured received signals when the DUT is not present

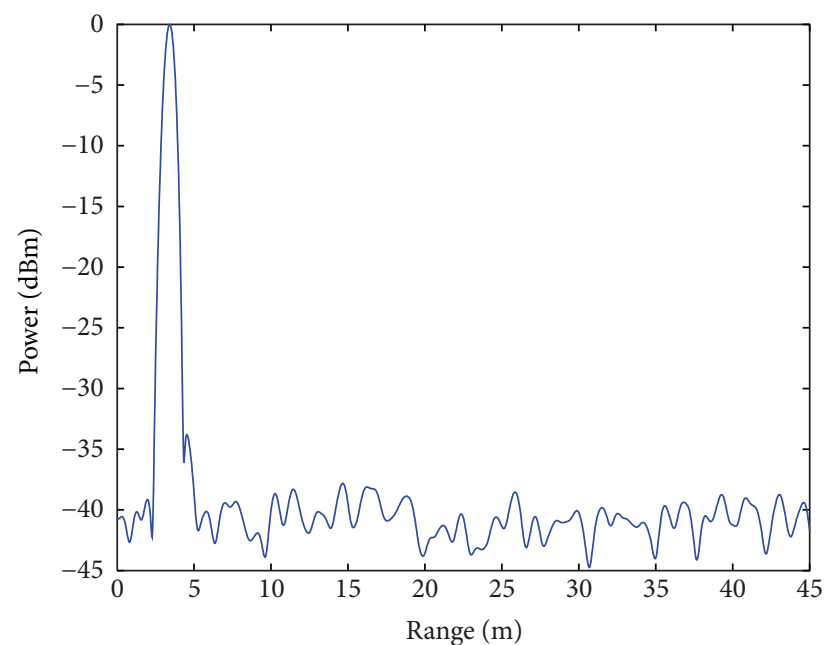

(b) Normalized range profile of the received signals. The range profile is estimated using the IFFT with zero padding and the Blackman-Harris Window function

FIGURE 5: Power spectrum and range resolution of the received signals in $S_{4}$ collected by the harmonic step-frequency radar measurement system.

the frequency band under consideration in calculating (15), which will very possibly reduce the SINR. That is, we are likely to encounter a larger number of RFI "spikes" as our bandwidth increases. The goal then of the proposed SS-MO technique is to maximize the conflicting objective functions of (15) and (16) using multiobjective optimization. Multiobjective optimization is formulated using the following linear weighting function:

$$
Z_{i, j}=\alpha Z^{\prime} 1_{i, j}+(1-\alpha) Z^{\prime} 2_{i, j}
$$

where $0 \leq \alpha \leq 1$ is the user-defined weighting parameter, $Z^{\prime} 1_{i, j}$ is the normalized objective function of $Z 1_{i, j}$, and $Z^{\prime} 2_{i, j}$ is the normalized objective function of $Z 2_{i, j}$. The goal of (17) is to adjust the input parameters $(i, j)$ (i.e., the knobs) such that $Z_{i, j}$ (the meter) is maximized. The solution to this optimization problem is solved using

$$
\left(i^{*}, j^{*}\right)=\underset{i, j}{\arg \max }\left(Z_{i, j}\right),
$$

subject to the constraints

$$
\begin{aligned}
& \frac{\operatorname{Pr}_{i, j}}{\widehat{\Gamma}_{i, j}} \geq Z 1_{\text {min }}, \\
& \frac{4 \beta_{i, j}}{c} \geq Z 2_{\text {min }} .
\end{aligned}
$$

These constraints are used to meet the minimum SINR and range resolution requirements of the radar. The chosen subband size is then determined using (11), $\beta^{*}=i^{*} \bar{F}_{\Delta}$, and the optimal subband center frequency is defined using (12), $f_{c}^{*}=\left[\bar{f}\left(j^{*}\right)+\bar{f}\left(j^{*}+i^{*}-1\right)\right] / 2$. The parameters $\left(\beta^{*}, f_{c}^{*}\right)$ are then fed back to the radar and used for waveform synthesis.

The computational complexity to form $Z_{i, j}$ in (17) requires $2 \dot{N}$ divisions (for normalizing both objective functions); $2 \dot{N}$ multiplications (i.e., multiplication by $\alpha$ ); and $\dot{N}$ summations (adding the objective functions). The total 
TABLE 1: Total computational complexity of the SS-MO technique.

\begin{tabular}{lccccc}
\hline Equation & Equation (10) & Equation (13) & Equation (15) & Equation (17) & Total \\
\hline Sums & $\left(N^{2}-N\right) / 2$ & 0 & 0 & $\dot{N}$ & $N^{2}$ \\
Multiplications/divisions & 0 & $\dot{N}-N$ & $\dot{N}$ & $4 \dot{N}$ & $3 N^{2}+2 N$ \\
\hline
\end{tabular}

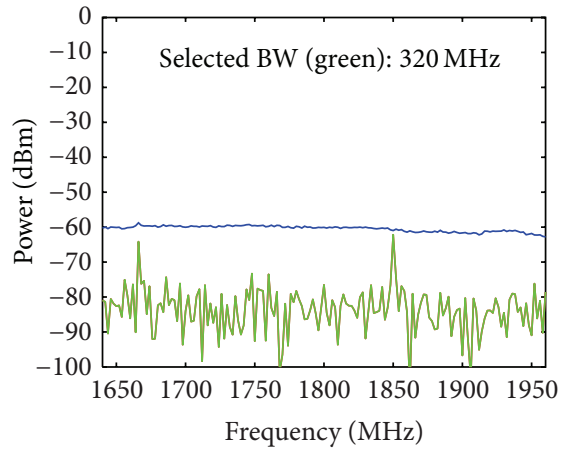

(a) RFI input power: $-95 \mathrm{dBm}$

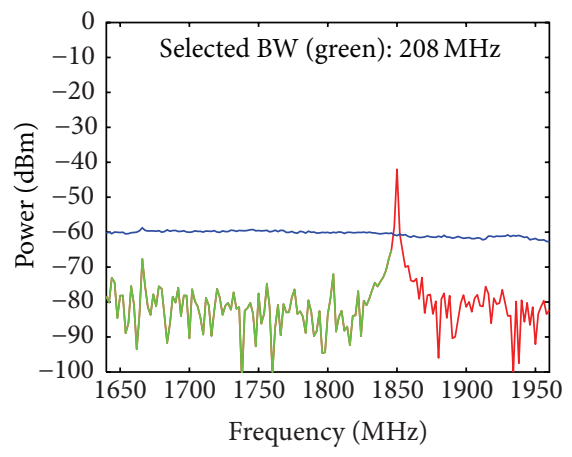

(d) RFI input power: $-75 \mathrm{dBm}$

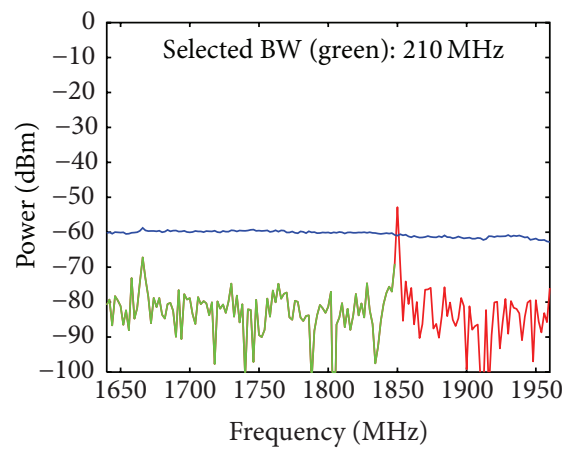

(b) RFI input power: $-85 \mathrm{dBm}$

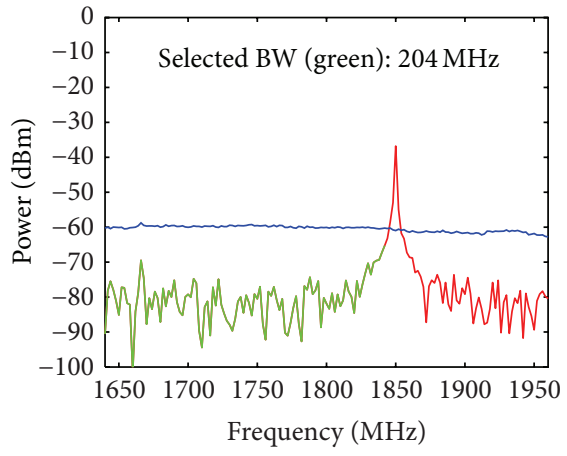

(e) RFI input power: $-70 \mathrm{dBm}$

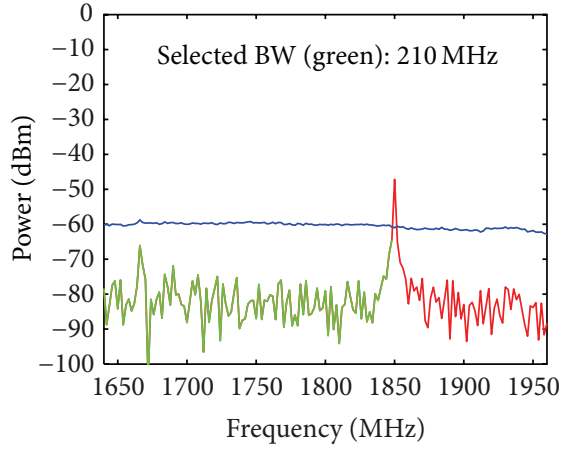

(c) RFI input power: $-80 \mathrm{dBm}$

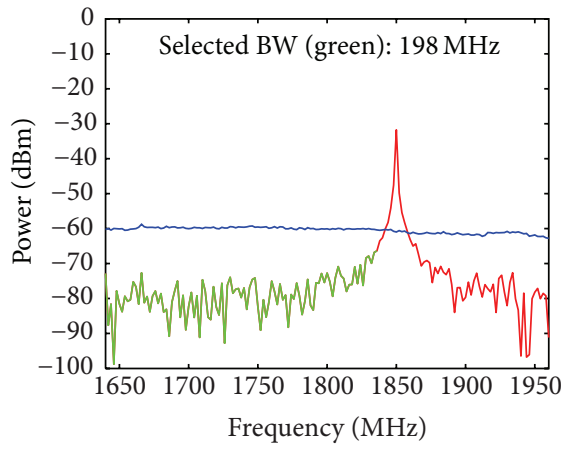

(f) RFI input power: $-65 \mathrm{dBm}$

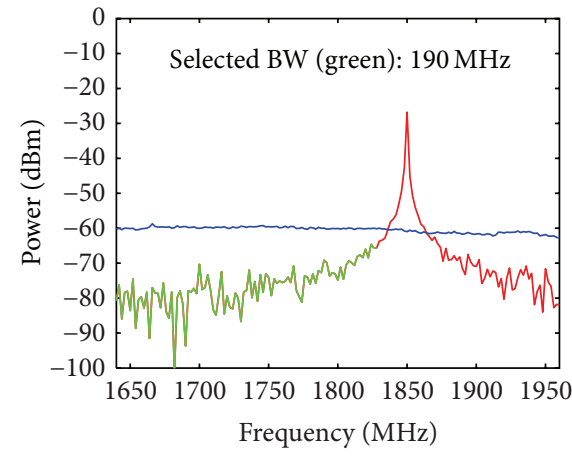

(g) RFI input power: $-60 \mathrm{dBm}$

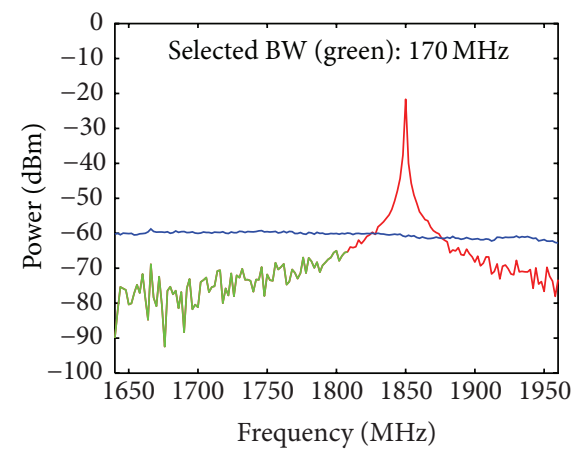

(h) RFI input power: $-55 \mathrm{dBm}$

FIGURE 6: Power spectra of the narrowband RFI. The blue line shows the target response (displayed for comparison with RFI). The red line shows the RFI. The green line is overlaid onto the red line and indicates the subband selected by the SS-MO technique.

complexity of the proposed SS-MO technique is summarized in Table 1.

\section{Experiment and Results}

In this section, experimental data, collected by a harmonic step-frequency radar measurement system, are analyzed using the proposed technique described in Section 3. The hardware architecture of the measurement system is specially designed to reject unwanted self-generated interference and produce highly linearized transmit waveforms. The experiments are used to probe a nonlinear circuit element that is directly connected to the radar (wired experiment). RFI is then injected into the radar receiver. Two types of RFI signals are considered: (1) narrowband interference with a fixed frequency that increases in amplitude and (2) wideband interference with fixed amplitude that increases in frequency. The proposed SS-MO technique independently processes the 
power spectrum of the RFI and selects a subband that solves the optimization criteria specified in (18). The measured SINR, range resolution cell size, and peak-to-sidelobe ratio are measured to quantify harmonic radar performance using the proposed SS-MO technique. These results are then compared with the performance of the harmonic radar operating at full bandwidth (without using the proposed technique).

4.1. Harmonic Step-Frequency Radar Measurement System. A block diagram of the harmonic step-frequency radar measurement system is shown in Figure 4. The hardware is controlled using custom-designed algorithms implemented on the National Instruments (NI) PXI-1056 chassis. The PXI1056 chassis controls two NI-5651 signal generators and a LeCroy Wave Master 8300a oscilloscope. The oscilloscope collects data at a rate of 5 Giga-Samples (GS) per second (GS/s) with 8 bits of resolution. The NI-5651 signal generator at point $(\mathrm{A})$ generates the step-frequency waveform that is split into a reference signal (to the oscilloscope) and a probe signal.

The probe signal is input into the transmitter circuit components starting from point (A) to point (B) (Figure 4). The probe signal is first filtered by two Mini-Circuits RDP$272+$ diplexers; the diplexers are connected via the low port and function as a low-pass filter. Diplexers are used because they have low insertion loss (less than $2 \mathrm{~dB}$ ) and low voltage standing wave ratio (VSWR) (less than 1.5 for the two diplexers in cascade) and they provide greater than $90 \mathrm{~dB}$ rejection of the self-generated harmonics. A second pair of diplexers, Reactel 2DP2P-900/1800, is used to provide additional attenuation (greater than $80 \mathrm{~dB}$ with only $0.4 \mathrm{~dB}$ of insertion loss) of the self-generated second harmonics. An isolator (with less than $5 \mathrm{~dB}$ of loss) connects these two diplexers and is used to provide attenuation of any signals reflected from the device under test (DUT). A $10 \mathrm{~dB}$ attenuator is used to prevent saturation of the DUT and to further reduce the self-generated harmonics. The transmitter circuit components described in this paragraph constitute LTI System 1 (Figure 1) with system response $H_{1}(\omega)$.

The DUT is a Mini-Circuits ZJL-4G+ amplifier and is not powered during experimentation. The amplifier serves as the nonlinear target of Figure 1. The response of the DUT is next reflected into the receiver chain of the harmonic step-frequency radar measurement system and propagates from point (B) to point (C). This signal first propagates sequentially through the $10 \mathrm{~dB}$ attenuator, the high port of the Reactel diplexer, a Western Microwave isolator (used to prevent undesirable reflections), and an Omni-Spectra 200G3 coupler. The Reactel diplexer functions as a highpass filter to attenuate the linear step-frequency waveform and preserve the harmonic response from the target. The coupler injects the RFI signal into the receiver. Three MiniCircuits diplexers are next used to attenuate the probe signal by dissipating it in $50 \mathrm{ohm}$ terminators and passing the harmonic target response with less than $3 \mathrm{~dB}$ loss. The probe signal must be attenuated since it could generate harmonics in the circuitry of the receiver. The response signal is next amplified using two Mini-Circuits ZRL-2400LNT Low Noise Amplifiers (LNAs) connected via a $3 \mathrm{~dB}$ attenuator to prevent

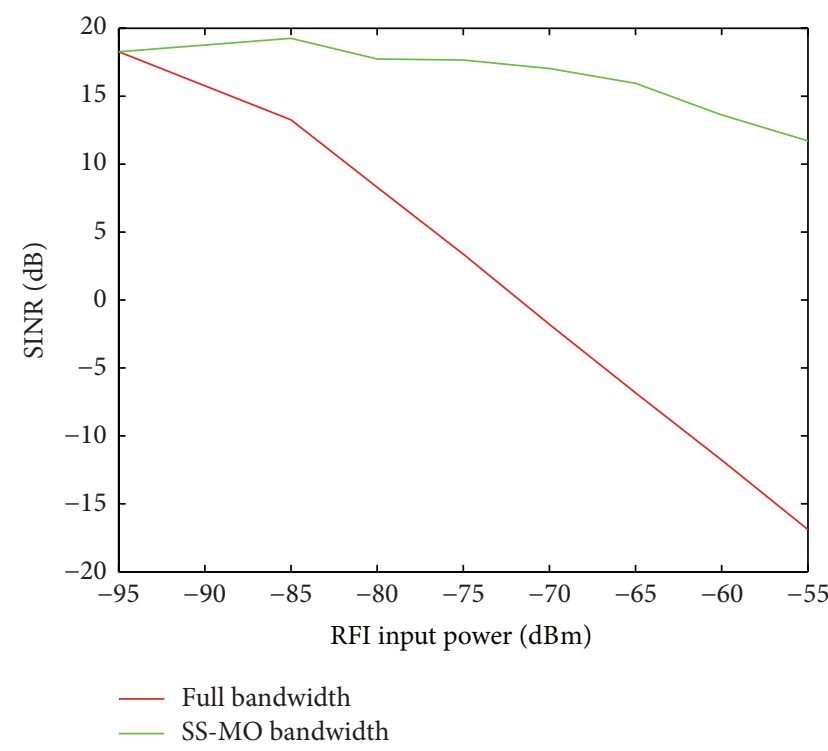

FIGURE 7: The SINR results of the narrowband RFI experiment. These results indicate that the SS-MO technique significantly improves SINR when high power, narrowband interference is present in band to the harmonic step-frequency radar.

ringing and improve matching. Two additional Mini-Circuits diplexers are used to further reduce the power of the probe signal and allow only the harmonic response of the DUT to be digitized. The transmitter circuit components described in this paragraph constitute LTI System 2 (see Figure 1) with system response $\mathrm{H}_{2}(\omega)$.

The harmonic step-frequency radar measurement system is used to collect data from the DUT with added RFI. The measurement system probes the DUT from 820 to $980 \mathrm{MHz}$ with a frequency step size of $1 \mathrm{MHz}$ and amplitude $\Lambda_{1}=$ 0.2236 Volts $(\mathrm{V})$ RMS $(0 \mathrm{dBm})$ to produce $N=160$ stepfrequency probe signals. The total transmitter gain, from point (A) to point (B), is $\left|H_{1}\right|=0.1778$. Based on S11, that is, input reflection coefficient measurements of the DUT, the second order power series coefficient is calculated as $a_{2}=0.0025$. The total receiver gain, from point $(\mathrm{B})$ to point (C), is $\left|H_{2}\right|=56.23$. The received signals are denoted by $S_{4}=\left\{S_{4}(1, t), \ldots, S_{4}(160, t)\right\}$ and the amplitudes are estimated using (5): $\Lambda_{4}=a_{2} \Lambda_{1}\left|H_{1}\right|^{2}\left|H_{2}\right|=0.224 \mathrm{mV}$ RMS corresponding to a power of $P_{4}=\Lambda_{4}^{2} / \Omega=1 \mathrm{nW}(-60 \mathrm{dBm})$, where $\Omega=50$. The frequencies of the received signals are $\bar{f}(n)=\{1640 \mathrm{MHz}, \ldots, 1960 \mathrm{MHz}\}$ with a frequency step size $\bar{F}_{\Delta}=2 \mathrm{MHz}$ and occupy a bandwidth of $\bar{B}=320 \mathrm{MHz}$. The frequency resolution cell size at full bandwidth is $R_{\text {res }}=$ $0.3125 \mathrm{~m}$ from (8) with $c=2 \times 10^{8}(\mathrm{~m} / \mathrm{s})$; note that $c$ is the speed of propagation in a cable [33]. The computational complexity for this experiment is insignificant since the total number of summations is equal to 25,600 and the total number of multiplications/divisions is equal to 77,120 (using Table 1 with $N=160$ ). However, alternative formulations of the proposed technique, such as those discussed in [34], should be considered for radar applications that require a high frequency resolution. 


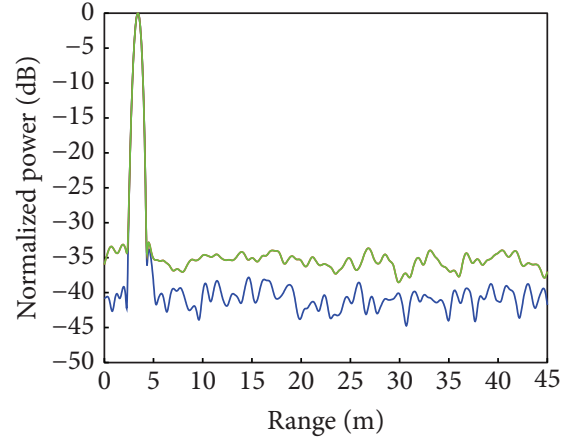

(a) RFI input power: $-95 \mathrm{dBm}$

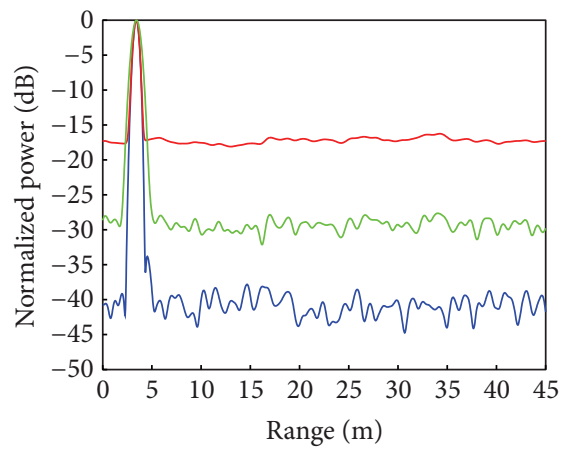

(d) RFI input power: $-75 \mathrm{dBm}$

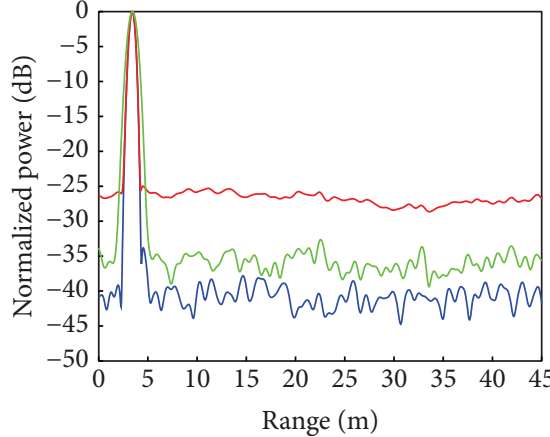

(b) RFI input power: $-85 \mathrm{dBm}$

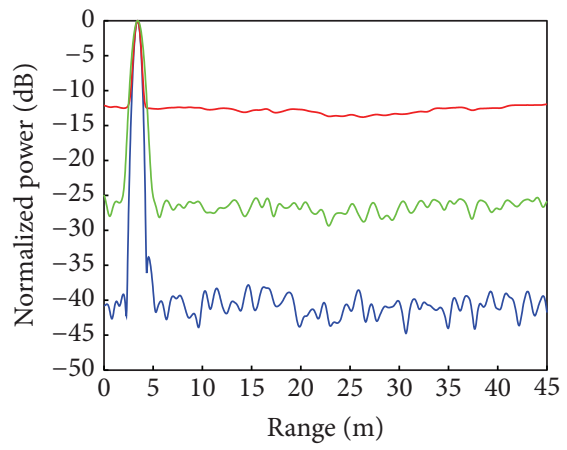

(e) RFI input power: $-70 \mathrm{dBm}$

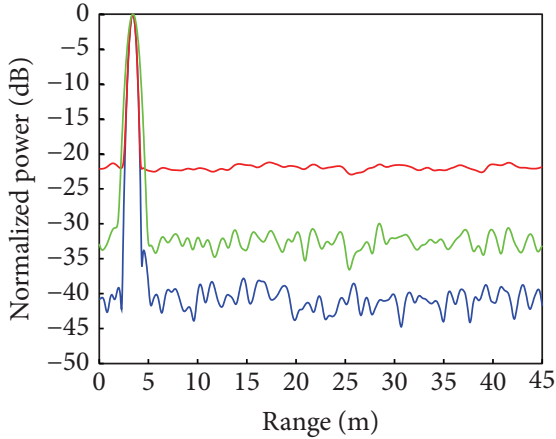

(c) RFI input power: $-80 \mathrm{dBm}$

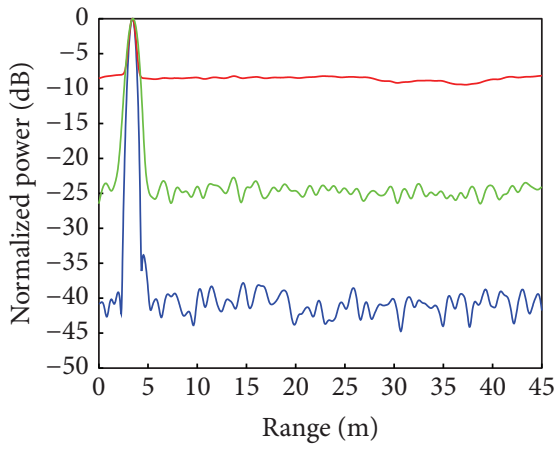

(f) RFI input power: $-65 \mathrm{dBm}$

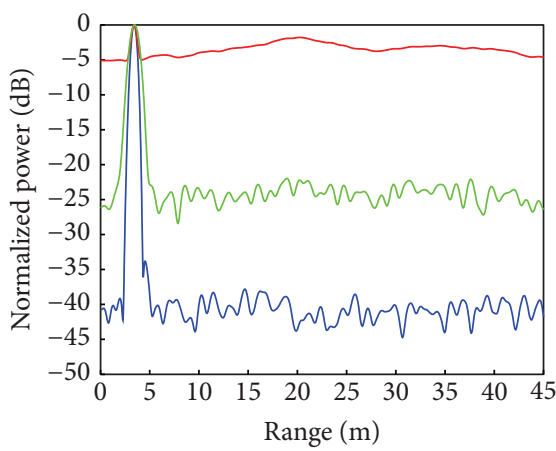

(g) RFI input power: $-60 \mathrm{dBm}$

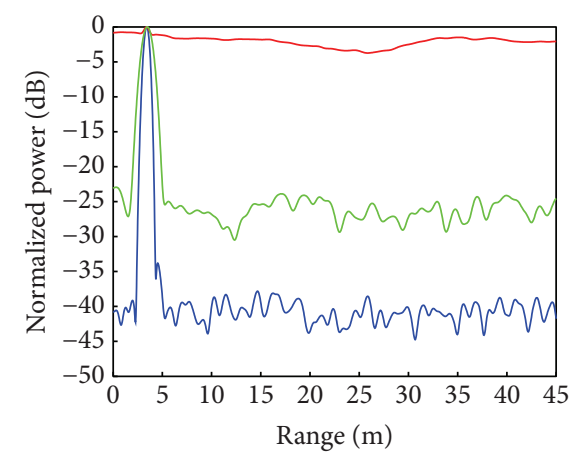

(h) RFI input power: $-55 \mathrm{dBm}$

FIGURE 8: Range profiles for the narrowband RFI experiment. As shown, the narrowband RFI significantly increases sidelobe power as compared to the sidelobe power generated by the SS-MO technique (due to the reduced bandwidth).

The measured received signals are shown in Figure 5(a). Two plots are shown in the figure and illustrate (1) the power spectrum of the measured received signals when the DUT is present (green plot) and (2) the power spectrum of the measured received signals when the DUT is not present (blue plot), with a noise power of $-80 \mathrm{dBm}$. Observe that the response of the DUT is relatively flat across the band (less than $3 \mathrm{~dB}$ of fluctuations) and $15 \mathrm{~dB}$ above the noise floor. The normalized range profile of the received signals is illustrated in Figure 5(b). The range profile is estimated using the IFFT with zero padding (10x interpolation) and windowing (Blackman-Harris Window). Figure 5(b) clearly displays the target at $3.41 \mathrm{~m}$ and sidelobes oscillating near $-40 \mathrm{dBm}$. It should be noted in the context of these experiments that the "range" measurement is directly proportional to the time that the probe signal propagates through the measurement system; further ranges could be observed in the range profile if a length of cable is used between the target and the measurement system. The target location of $3.41 \mathrm{~m}$ corresponds to a propagation delay from the transmitter to the receiver of $17.1 \mathrm{~ns}$.

4.2. Narrowband RFI Experiment. In the first experiment, narrowband RFI is injected into the receiver of the harmonic step-frequency radar measurement system at frequency $1860 \mathrm{MHz}$; the transmitter remains inactive with no DUT present in the measurement system. The measurement system is used to probe the DUT after the SS-MO technique analyzes the corrupted spectra. The power of the RFI increases from $-95 \mathrm{dBm}$ to $-55 \mathrm{dBm}$ in $5 \mathrm{~dB}$ steps and is amplified by the LNAs of Figure 4. This experiment produces 8 sets of received signals (one set per RFI power increase). The SS-MO 
technique is then used to process each set of received signals in order to find the subband $\beta^{*}$. The algorithm uses the following parameters: $Z 1_{\text {min }}=-20 \mathrm{~dB}, Z 2_{\min }=0.3125 \mathrm{~m}$, $Z 2_{\max }=3.33 \mathrm{~m}$, and $\alpha=0.5$. The value of $\alpha$ is selected to provide equal emphasis of both objective functions. The power spectra of the narrowband RFI are shown in Figure 6, where the green line indicates the subband selected by the SSMO technique. First observe how the noise floor is elevated at the frequencies surrounding the narrowband interference. The SS-MO technique appropriately avoids these elevated regions by reducing the subband.

The SINR results (using (15)) of the narrowband RFI experiment are illustrated in Figure 7. Two SINR estimates are measured: (1) the SINR for bandwidth $B$ denoted by $\mathrm{SINR}_{B}$ (red line) and (2) the SINR for the SS-MO technique with subband $\beta^{*}$ denoted by $\operatorname{SINR}_{\beta^{*}}$ (green line). Notice the significant reduction of SINR from $18.6 \mathrm{~dB}$ to $-16.6 \mathrm{~dB}$ for $\mathrm{SINR}_{B}$. In contrast, $\mathrm{SINR}_{\beta^{*}}$ maintains a higher level of SINR with a maximum difference of over $25 \mathrm{~dB}$ (at $-55 \mathrm{dBm}$ RFI input power in Figure 7). These results indicate that the SSMO technique significantly improves SINR when high power, narrowband interference is present in band to the harmonic step-frequency radar. By maintaining a high SINR using the SS-MO technique, it becomes more likely for the harmonic step-frequency radar to increase detection performance or lower the false alarm rate.

The harmonic step-frequency radar measurement system is next used to probe the DUT using the full bandwidth $B$ and the subband selected by the SS-MO algorithm $\beta^{*}$. The IFFT (with zero padding and windowing) is then used to process the received signals to estimate range profiles. These range profiles are shown in Figure 8. The blue lines represent the range profile when the target is present without RFI (the same as in Figure 5(b)); this range profile is the same in each subfigure. The red lines represent the range profiles using the full bandwidth with RFI present. Observe the great increase in sidelobe power caused by the increase in RFI power. The green lines represent the range profiles using the subbands selected by the SS-MO algorithm. Although an elevated sidelobe power is observed using the SS-MO technique (due to the reduced bandwidth), this power is significantly less than that produced by the RFI at full bandwidth.

The peak-to-average sidelobe ratio (PSLR) is used to quantify the sidelobe power levels in Figure 8 and is defined as

$$
\operatorname{PSLR}=\frac{P_{\text {peak }}}{P_{\text {SL }}},
$$

where $P_{\text {peak }}$ is the peak power of the DUT (at $3.41 \mathrm{~m}$ ), $P_{\mathrm{SL}}=$ $(1 / M) \sum_{m=1}^{M} \chi_{m}, \chi_{m} \in \chi$ is the $m$ th sidelobe power sample, $\chi$ is the set of all sidelobe power samples (or range bins) outside the first lower and upper null of the main lobe, and $M$ is the total number of samples. The PSLR for the range profiles of Figure 8 are shown in Figure 9. The blue line represents the PSLR when the target is present without RFI. The red line represents the PSLR using the full bandwidth with RFI present. The green line represents the PSLR using

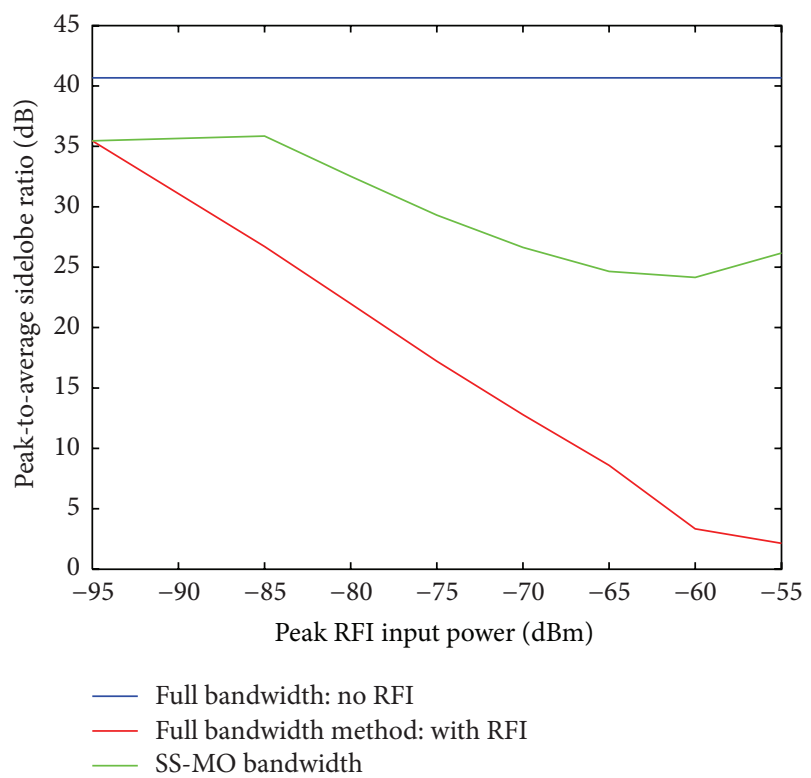

FIGURE 9: The PSLR results of the narrowband RFI experiment. The PSLR measured for the SS-MO algorithm is maintained at a higher level as the narrowband interference power increases.

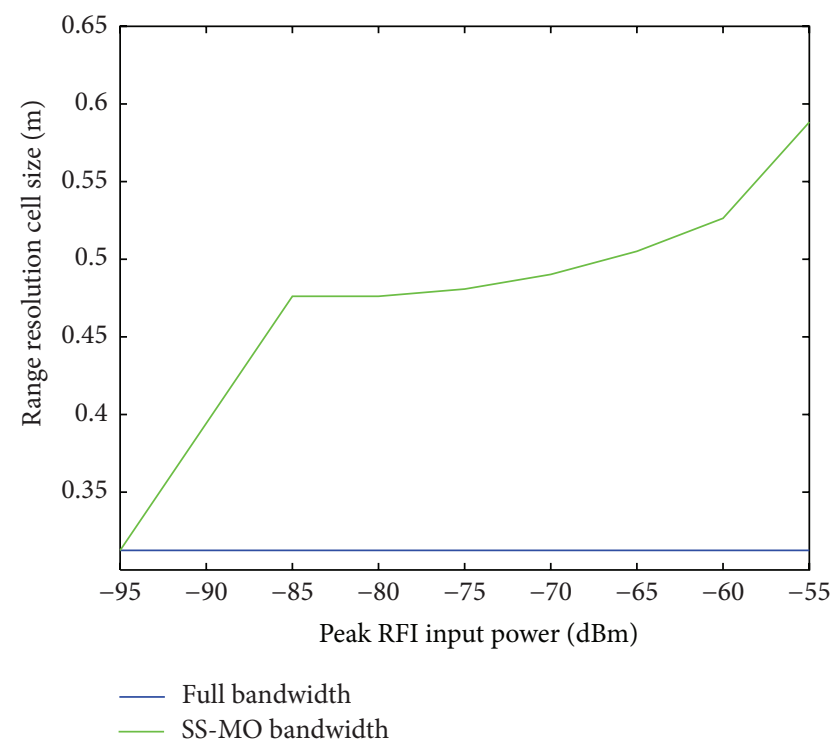

FIGURE 10: The range resolution cell size results for the narrowband RFI experiment. As is shown, the range resolution cell size is increased when using the SS-MO technique but is within the maximum cell size boundary condition specified by $Z 2_{\max }=3.33 \mathrm{~m}$.

the subbands selected by the SS-MO algorithm. Notice the significant reduction of PSLR from $35.45 \mathrm{~dB}$ to $2.14 \mathrm{~dB}$ using the full bandwidth (i.e., red line). In contrast, the PSLR measured for the SS-MO algorithm is significantly higher with a maximum increase of over $25 \mathrm{~dB}$ (at an RFI input power level of $-55 \mathrm{dBm}$ in Figure 9). These results indicate that the SS-MO technique significantly improves PSLR when high power, narrowband interference is present in band to 


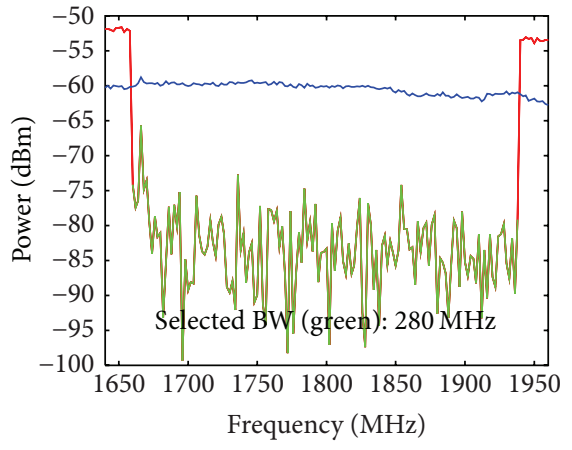

(a) RFI bandwidth: $40 \mathrm{MHz}$

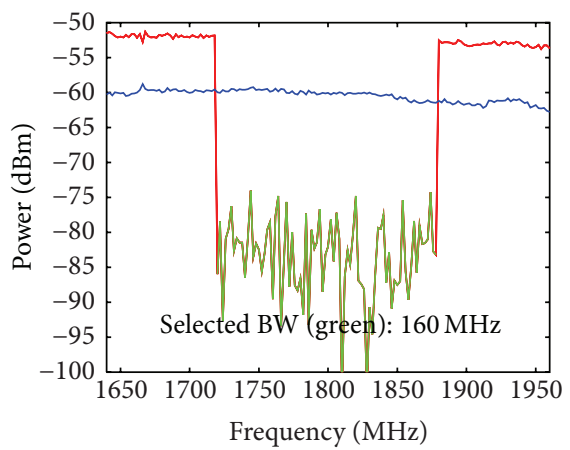

(d) RFI bandwidth: $160 \mathrm{MHz}$

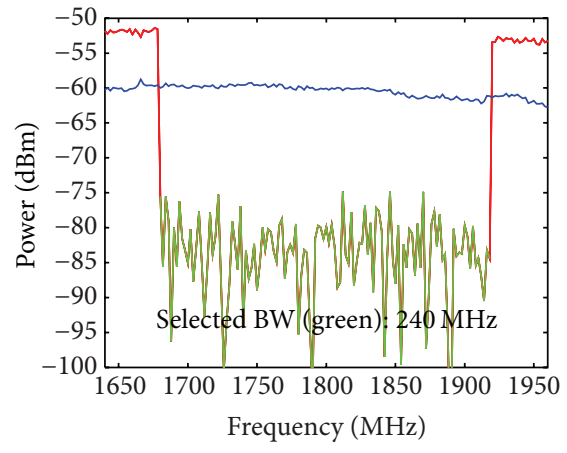

(b) RFI bandwidth: $80 \mathrm{MHz}$

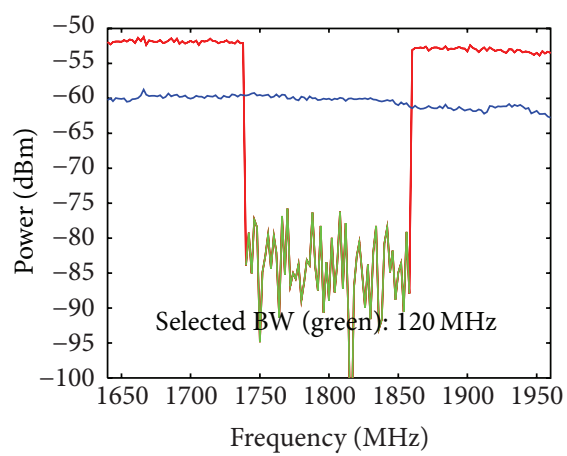

(e) RFI bandwidth: $200 \mathrm{MHz}$

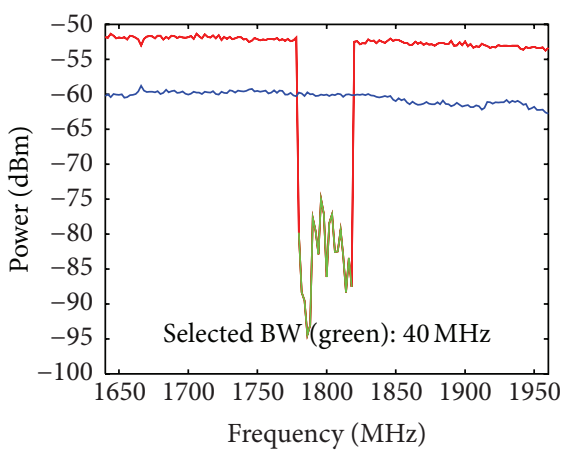

(g) RFI bandwidth: $280 \mathrm{MHz}$

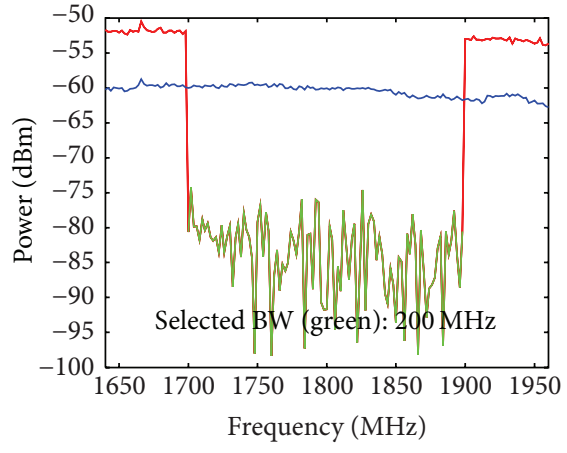

(c) RFI bandwidth: $120 \mathrm{MHz}$

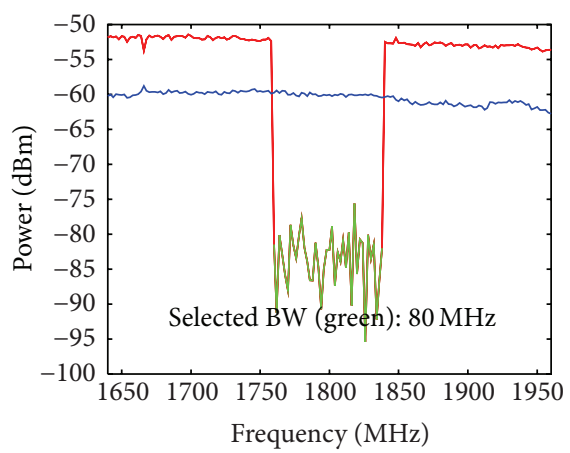

(f) RFI bandwidth: $240 \mathrm{MHz}$

FIgURE 11: Power spectra of the wideband RFI. The blue line shows the target response (displayed for comparison with RFI). The red line shows the RFI. The green line is overlaid onto the red line and indicates the subband selected by SS-MO.

the harmonic step-frequency radar. By maintaining a high PSLR using the SS-MO technique, it becomes more likely for the harmonic radar to detect responses from other nonlinear targets at different ranges (other than the DUT). If the SS-MO technique is not used, then responses from other nonlinear targets (at different ranges) would be masked by the high sidelobe power caused by the RFI. Hence, the SSMO technique offers a capability to better detect additional nonlinear targets at different ranges.

The final metric considered in this analysis is the range resolution cell size. The measured range resolution cell size is shown in Figure 10. The red line represents the range resolution cell size using the full bandwidth with RFI present calculated using $c /(2 \bar{B})$. The green line represents the range resolution cell size $c /\left(2 \beta^{*}\right)$ using the subbands selected by the SS-MO algorithm. As is shown, the range resolution cell size is increased when using the SS-MO technique but is within the maximum cell size boundary condition specified by $Z 2_{\max }=3.33 \mathrm{~m}$. As a consequence, it would be more challenging for the harmonic step-frequency radar to (1) separate closely spaced targets and (2) extract high fidelity features used to classify targets (i.e., target information is potentially reduced). Please note that a sharp transition in range resolution cell size is observed in Figure 10 when the input RFI power is increased from $-95 \mathrm{dBm}$ to $-85 \mathrm{dBm}$. At $-85 \mathrm{dBm}$ the algorithm avoids the RFI by "splitting" the bandwidth in two and selecting the left side of the spectrum (as shown in Figure 6(b)) resulting in a large initial loss of bandwidth (a loss of $110 \mathrm{MHz}$ ). Hence, the sharp transition observed in Figure 10 is caused by the initial loss of bandwidth. 


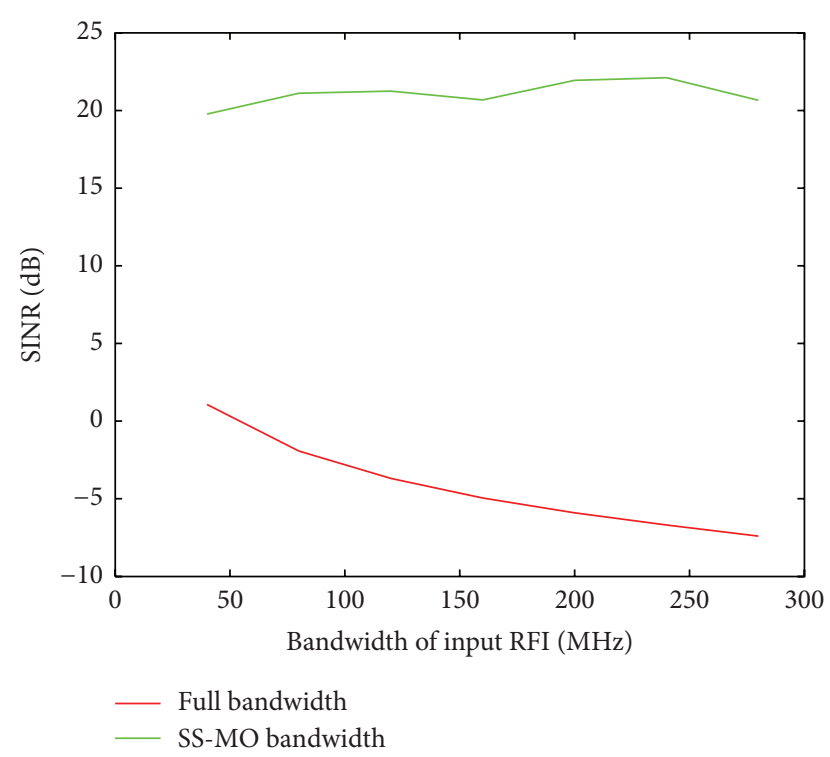

FIGURE 12: The SINR results of the wideband RFI experiment.

4.3. Wideband RFI Experiment. In the second experiment, wideband RFI is injected into the receiver of the harmonic step-frequency radar measurement system at frequency at power level of $-88 \mathrm{dBm}$ (and amplified by the LNAs of Figure 4); the transmitter remains inactive with no DUT present in the measurement system. The measurement system is used to probe the DUT after the SS-MO technique analyzes the corrupted spectra. The bandwidth of the RFI increases symmetrically with a total bandwidth ranging from $40 \mathrm{MHz}$ to $280 \mathrm{MHz}$ in $40 \mathrm{MHz}$ steps. This experiment produces 7 sets of received signals (one set per bandwidth increase). The SSMO technique, using the same parameters as in Section 4.1, is then used to process each set of received signals in order to find the subband $\beta^{*}$. The power spectra of the wideband RFI are shown in Figure 11, where the green line indicates the subband selected by the SS-MO technique. Observe that the RFI is increased inward from the upper and lower band. Similar to the results of the narrowband RFI experiments, the SS-MO technique appropriately avoids these elevated regions of RFI by reducing the subband size, thereby maintaining a high SINR.

The SINR results for the wideband RFI experiment are illustrated in Figure 12. Notice the significant reduction of SINR from $1 \mathrm{~dB}$ to $-7.4 \mathrm{~dB}$ when using the full bandwidth. In contrast, the SINR for SS-MO is significantly higher with a maximum difference of over $25 \mathrm{~dB}$ (at $280 \mathrm{MHz}$ input RFI bandwidth in Figure 12). Similar to the SINR results for the narrowband RFI experiment, the SS-MO technique significantly improves SINR when wideband RFI is present in band to the harmonic step-frequency radar. By maintaining a high SINR using the SS-MO technique, it becomes more likely for the harmonic step-frequency radar to increase detection performance or lower the false alarm rate.

The harmonic step-frequency radar measurement system is next used to probe the DUT using the full bandwidth $B$ and the subband selected by the SS-MO algorithm $\beta^{*}$. The IFFT is then used to process the received signals to estimate range profiles. These range profiles are shown in Figure 13. The blue lines represent the range profile when the target is present without RFI (the same as in Figure 5(b)); this range profile is the same in each subfigure. The red lines represent the range profiles using the full bandwidth with RFI present. Observe that the sidelobe power levels between the red and green lines are very similar when the RFI bandwidth is $40-80 \mathrm{MHz}$. The sidelobe power increase for the red line is caused by the RFI and the sidelobe power increase for the blue line is caused by the reduced bandwidth. When the RFI bandwidth is greater than $120 \mathrm{MHz}$, a large increase of sidelobe power is observed for the red line (caused by the RFI). Although an elevated sidelobe power is observed using the SS-MO technique (due to the reduced bandwidth), this power is significantly less than that produced by the RFI.

PSLR results are shown in Figure 14. As discussed in the previous paragraph, the sidelobe power levels are very similar when the RFI bandwidth is $40 \mathrm{MHz}$ and $80 \mathrm{MHz}$; this similarity is observed for PSLR. A significant increase of PSLR is observed for the SS-MO technique when the RFI bandwidth is greater than $80 \mathrm{MHz}$, with a maximum increase of over $15 \mathrm{~dB}$ (at an RFI bandwidth of $280 \mathrm{MHz}$ as shown in Figure 14). These results suggest that the SS-MO technique significantly improves PSLR when wideband interference (greater than $80 \mathrm{MHz}$ ) is present in band to the harmonic step-frequency radar. By maintaining a high PSLR using the SS-MO technique, it becomes more likely for the harmonic radar to detect responses from other nonlinear targets at different ranges (other than the DUT). If the SS-MO technique is not used, then responses from other nonlinear targets (at different ranges) would be masked by the high sidelobe power caused by the RFI. Hence, the SS-MO technique offers a capability to better detect additional nonlinear targets at different ranges.

The final metric considered in this analysis is the range resolution cell size. The measured range resolution cell size is shown in Figure 15. The red line represents the range resolution cell size using the full bandwidth with RFI present calculated using $c /(2 \bar{B})$. The green line represents the range resolution cell size $c /\left(2 \beta^{*}\right)$ using the subbands selected by the SS-MO algorithm. As is shown, the range resolution cell size is increased when using the SS-MO technique but is within the maximum cell size boundary condition specified by $Z 2_{\max }=3.33 \mathrm{~m}$. As a consequence, it would be more challenging for the harmonic step-frequency radar to (1) separate closely spaced targets and (2) extract high fidelity features used to classify targets (i.e., target information is potentially reduced).

\section{Conclusion}

In this paper, the SS-MO technique was used for harmonic step-frequency radar. This technique passively monitors the RF spectrum for subbands of high SINR within a constrained bandwidth of interest. An optimal subband is selected for the harmonic radar that maximizes SINR and minimizes the range resolution cell size, two conflicting objectives. The results in Section 4, for both deterministic narrowband and 


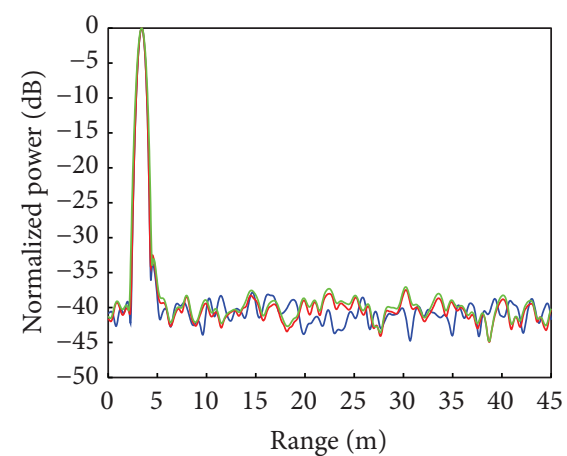

(a) RFI bandwidth: $40 \mathrm{MHz}$

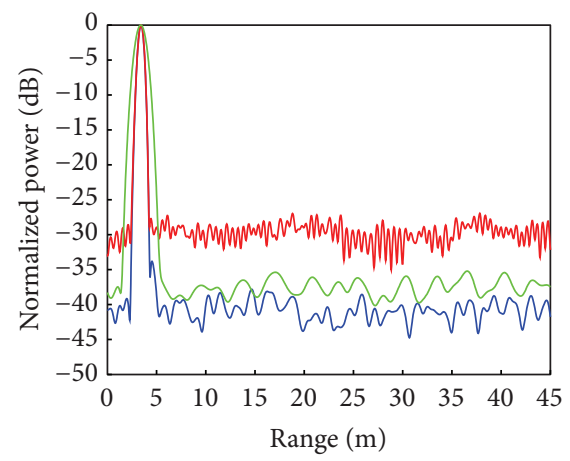

(d) RFI bandwidth: $160 \mathrm{MHz}$

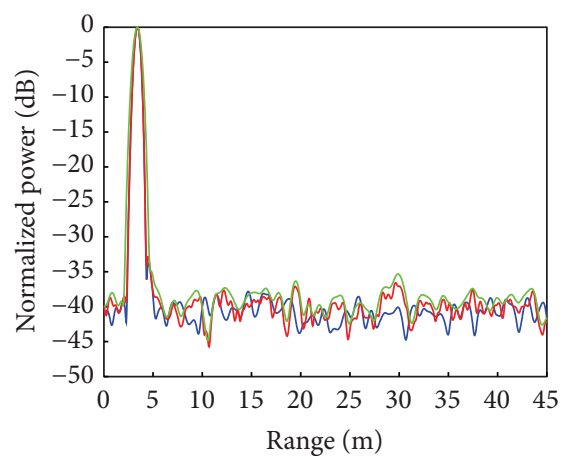

(b) RFI bandwidth: $80 \mathrm{MHz}$

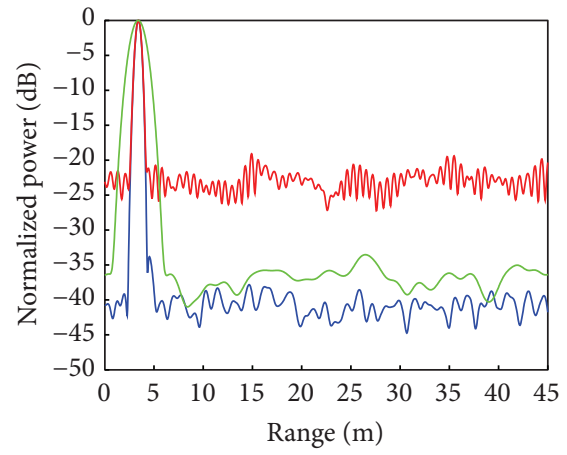

(e) RFI bandwidth: $200 \mathrm{MHz}$

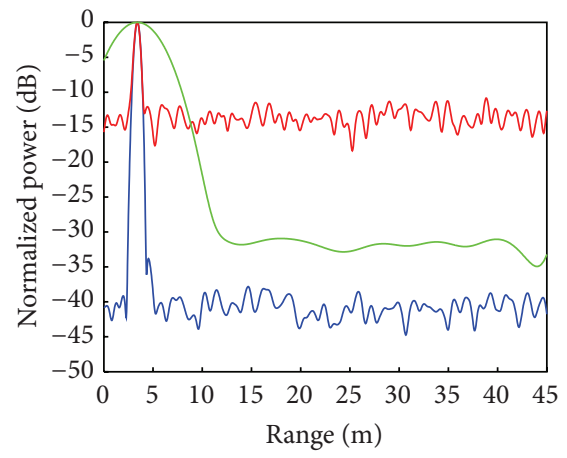

(g) RFI bandwidth: $280 \mathrm{MHz}$

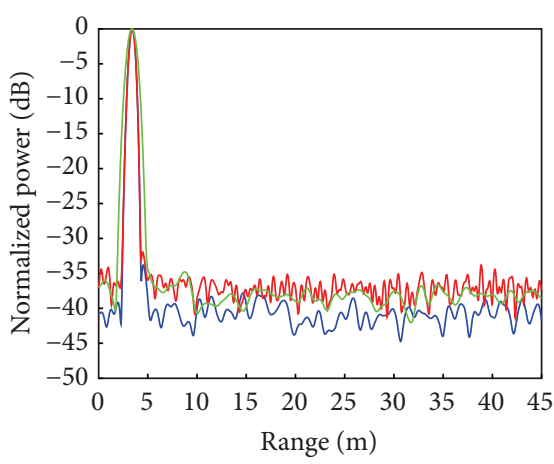

(c) RFI bandwidth: $120 \mathrm{MHz}$

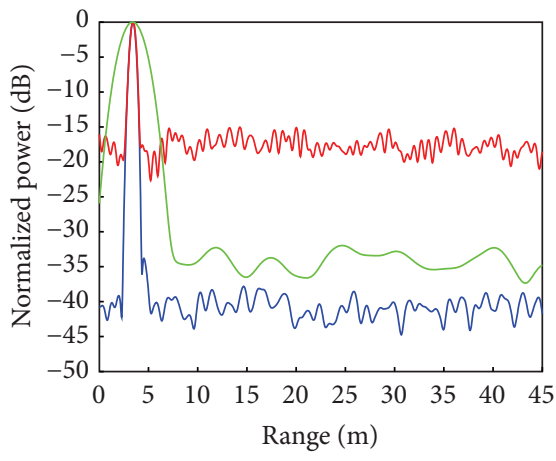

(f) RFI bandwidth: $240 \mathrm{MHz}$

FIGURE 13: Range profiles for the wideband RFI experiment. As shown, the wideband RFI significantly increases sidelobe power as compared to the sidelobe power generated by the SS-MO technique (due to the reduced bandwidth).

wideband interference, indicated that the SS-MO technique (1) significantly increases SINR by over $25 \mathrm{~dB}$ and (2) significantly increases the PSLR by over $15 \mathrm{~dB}$. By maintaining a high SINR using the SS-MO technique, it becomes more likely for the harmonic step-frequency radar to increase detection performance or lower the false alarm rate. By maintaining a high PSLR using the SS-MO technique, it becomes more likely for the harmonic radar to detect responses from other nonlinear targets at different ranges (other than the DUT). The disadvantage of the SS-MO technique for harmonic step-frequency radar is that the range resolution cell size is increased, which could lead to an inability for the radar to (1) separate closely spaced targets and (2) extract high fidelity features used to classify targets (i.e., target information is potentially reduced).

It should be noted that the proposed technique is designed to find a single frequency subband with high SINR within the overall bandwidth of the radar. It is reasonable to extend this design to find additional subbands with high SINR. The problem then becomes that of combining multiple discontinuous subbands to maximize the available bandwidth. Future work will include development of methods to combine these multiple subbands. Other future work will include time-frequency analysis to address the challenge of nonstationary RFI. This work will include (1) spectrum monitoring analysis to better understand and characterize time 


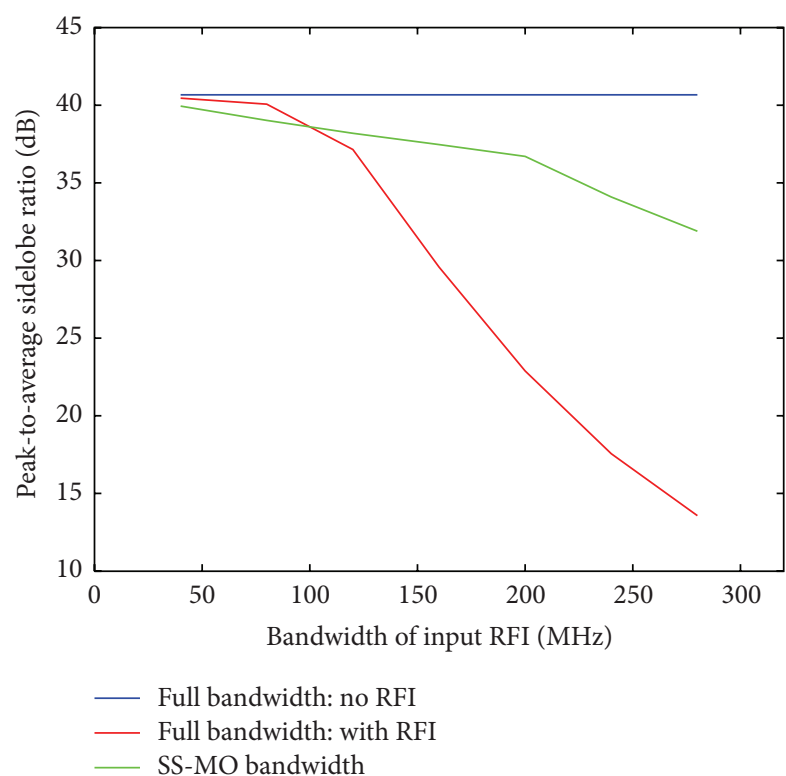

FIgURE 14: The PSLR results of the wideband RFI experiment. The PSLR measured for the SS-MO algorithm is maintained at a higher level as the narrowband interference power increases.

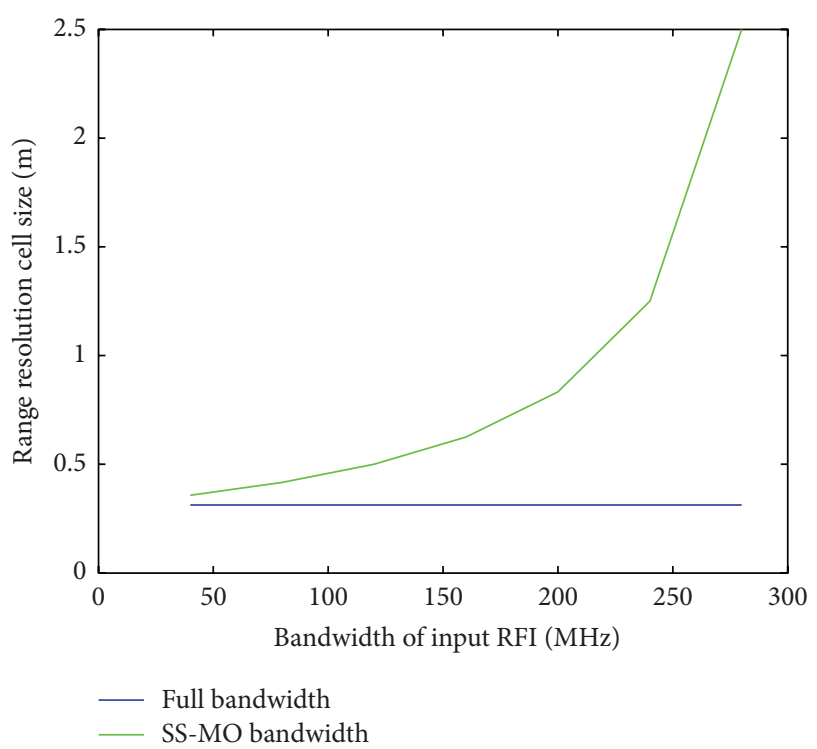

FIGURE 15: The range resolution cell size results for the wideband RFI experiment. As is shown, the range resolution cell size is increased when using the SS-MO technique but is within the maximum cell size boundary condition specified by $Z 2_{\max }=3.33 \mathrm{~m}$.

varying aspects of RFI and (2) investigation of methods to increase radar performance including techniques on random frequency selection and learning.

\section{Conflict of Interests}

The authors declare that there is no conflict of interests regarding the publication of this paper.

\section{References}

[1] A. F. Martone, "Cognitive radar demystified," Radio Science Bulletin, no. 350, pp. 10-22, 2014.

[2] H. Griffiths, L. Cohen, S. Watts et al., "Radar spectrum engineering and management: technical and regulatory issues," Proceedings of the IEEE, vol. 103, no. 1, pp. 85-102, 2015.

[3] F. H. Sanders, R. L. Sole, B. L. Bedford, D. Franc, and T. Pawlowitz, "Effects of RF interference on radar receivers," NTIA Report 06-444, U.S. Department of Commerce, 2006.

[4] F. H. Sanders, R. L. Sole, J. E. Carroll, G. S. Secrest, and T. L. Allmon, "Analysis and resolution of RF interference to radars operating in the band $2700-2900 \mathrm{MHz}$ from broadband communication transmitters," NTIA Report 13-490, U.S. Department of Commerce, 2012.

[5] W. Z. Wang, M. Ganley, B. Randhawa, and I. Parker, "Interference from radars into adjacent band UMTS and WiMAX systems," ERA Report 2007-0554, Cobham Technical Services (CTS), Ofcom, 2007.

[6] T. Li, H. Yang, and Z.-O. Zhou, "RFI suppression based on phase-coded stepped-frequency waveform in through-wall radar," IEEE Transactions on Geoscience and Remote Sensing, vol. 53, no. 3, pp. 1583-1591, 2015.

[7] J. R. Piepmeier, J. T. Johnson, P. N. Mohammed et al., "Radiofrequency interference mitigation for the soil moisture active passive microwave radiometer," IEEE Transactions on Geoscience and Remote Sensing, vol. 52, no. 1, pp. 761-775, 2014.

[8] G. J. Mazzaro, A. F. Martone, and D. M. McNamara, "Detection of RF electronics by multitone harmonic radar," IEEE Transactions on Aerospace and Electronic Systems, vol. 50, no. 1, pp. 477490, 2014.

[9] T. Higgins, T. Webster, and A. K. Shackelford, "Mitigating interference via spatial and spectral nulling," IET Radar, Sonar \& Navigation, vol. 8, no. 2, pp. 84-93, 2014.

[10] K. Gerlach, "Thinned spectrum ultrawideband waveforms using stepped-frequency polyphase codes," IEEE Transactions on Aerospace and Electronic Systems, vol. 34, no. 4, pp. 13561361, 1998.

[11] Federal Communication Commission, "National Broadcast Plan," http://www.broadband.gov/.

[12] The White House, "Unleashing the Wireless Broadband Revolution," http://www.whitehouse.gov/the-press-office/presidentialmemorandum-unleashing-wireless-broadband-revolution/.

[13] A. F. Martone and E. J. Delp, "Characterization of RF devices using two-tone probe signals," in Proceedings of the IEEE/SP 14th Workshop on Statistical Signal Processing (SSP '07), pp. 161-165, IEEE, Madison, Wis, USA, August 2007.

[14] R. O. Harger, "Harmonic radar systems for near-ground infoliage nonlinear scatterers," IEEE Transactions on Aerospace and Electronic Systems, vol. 12, no. 2, pp. 230-245, 1976.

[15] G. M. Low, S. Morissette, R. G. Sea, and M. J. Frazier, "Junction range finder," U. S. Patent 3,732,567, May 8, 1973.

[16] P. Lui, "Passive intermodulation interference in communication systems," Electronics \& Communications Engineering Journal, vol. 2, no. 3, pp. 109-118, 1990.

[17] J. L. Osborne, S. J. Clark, R. J. Morris et al., "A landscapescale study of bumble bee foraging range and constancy, using harmonic radar," Journal of Applied Ecology, vol. 36, no. 4, pp. 519-533, 1999.

[18] C. Fazi, F. Crowne, and M. Ressler, Design Considerations for Nonlinear Scattering, ARL-TR-5684, US Army Research Laboratory, Adelphi, Md, USA, 2011. 
[19] K. A. Gallagher, R. M. Narayanan, G. J. Mazzaro, K. I. Ranney, A. F. Martone, and K. D. Sherbondy, "Moving target indication with nonlinear radar," in Proceedings of the IEEE International Radar Conference, Arlington, Va, USA, May 2015.

[20] S. R. J. Axelsson, "Analysis of random step frequency radar and comparison with experiments," IEEE Transactions on Geoscience and Remote Sensing, vol. 45, no. 4, pp. 890-904, 2007.

[21] A. Reigber and L. Ferro-Famil, "Interference suppression in synthesized SAR images," IEEE Geoscience and Remote Sensing Letters, vol. 2, no. 1, pp. 45-49, 2005.

[22] Z. Hao and W. Biyang, "Radio frequency interference suppression in small-aperture high-frequency radars," IEEE Geoscience and Remote Sensing Letters, vol. 9, no. 4, pp. 788-792, 2012.

[23] Y. Jun, W. Biyang, and W. Shicai, "Method to suppress radiofrequency interference in HF radars," Electronics Letters, vol. 40, no. 2, pp. 145-146, 2004.

[24] C. A. Jackson, J. R. Holloway, R. Pollard et al., "Spectrally efficient radar systems in the L and S bands," in Proceedings of the 2007 IET International Conference on Radar Systems, pp. 15-18, Edinburgh, UK, October 2007.

[25] A. Khawar, A. Abdel-Hadi, and T. C. Clancy, "Spectrum sharing between S-band radar and LTE cellular system: a spatial approach," in Proceedings of the IEEE International Symposium on Dynamic Spectrum Access Networks (DYSPAN '14), pp. 7-14, McLean, Va, USA, April 2014.

[26] S. S. Bhat, R. M. Narayanan, and M. Rangaswamy, "Bandwidth sharing and scheduling for multimodal radar with communications and tracking," IETE Journal of Research, vol. 59, no. 5, pp. 551-562, 2013.

[27] A. F. Martone, K. D. Sherbondy, K. I. Ranney, and T. V. Dogaru, "Passive sensing for adaptable radar bandwidth," in Proceedings of the IEEE International Radar Conference (RadarCon '15), Arlington, Va, USA, May 2015.

[28] M. A. Richards, J. A. Scheer, and W. A. Holm, Principles of Modern Radar, SciTech Publishing, Edison, NJ, USA, 1st edition, 2010.

[29] J. C. Pedro and N. B. Carvalho, "Nonlinear analysis techniques for distortion prediction," in Intermodulation Distortion in Microwave and Wireless Circuits, p. 82, Artech House, Boston, Mass, USA, 2003.

[30] A. T. Paulose, High radar range resolution with the step frequency waveform [M.S. thesis], Department of Electrical and Computer Engineering, Naval Postgraduate School, Monterey, Calif, USA, 1994.

[31] H. Urkowitz, "Energy detection of unknown deterministic signals," Proceedings of the IEEE, vol. 55, no. 4, pp. 523-531, 1967.

[32] M. F. Aburdene and T. J. Goodman, "The discrete Pascal transform and its applications," IEEE Signal Processing Letters, vol. 12, no. 7, pp. 493-495, 2005.

[33] M. B. Steer, “Transmission lines," in Microwave and RF Design: A System Approach, SciTech Publishing, 2010.

[34] A. F. Martone and K. I. Ranney, "Fast technique for wideband spectrum sensing," in Proceedings of the IEEE Antennas and Propagation Society International Symposium (APSURSI '14), pp. 1206-1207, IEEE, Memphis, Tenn, USA, July 2014. 

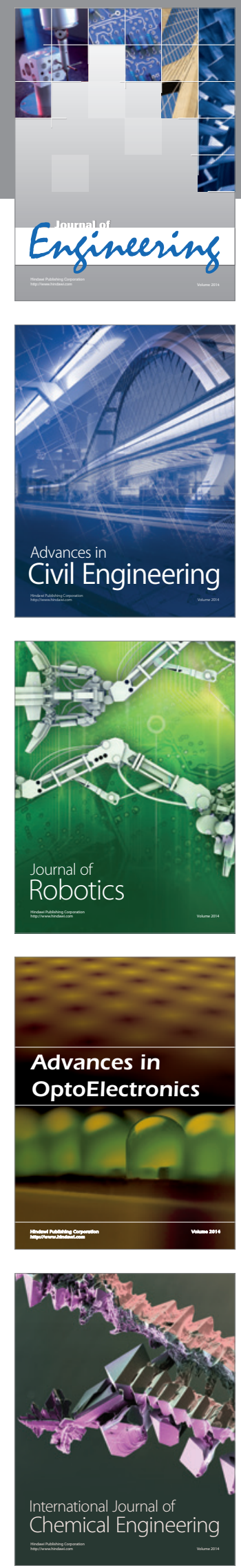

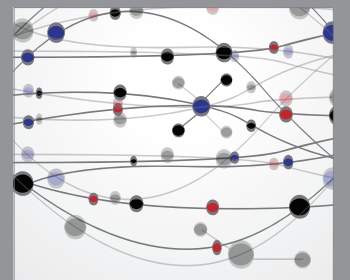

The Scientific World Journal
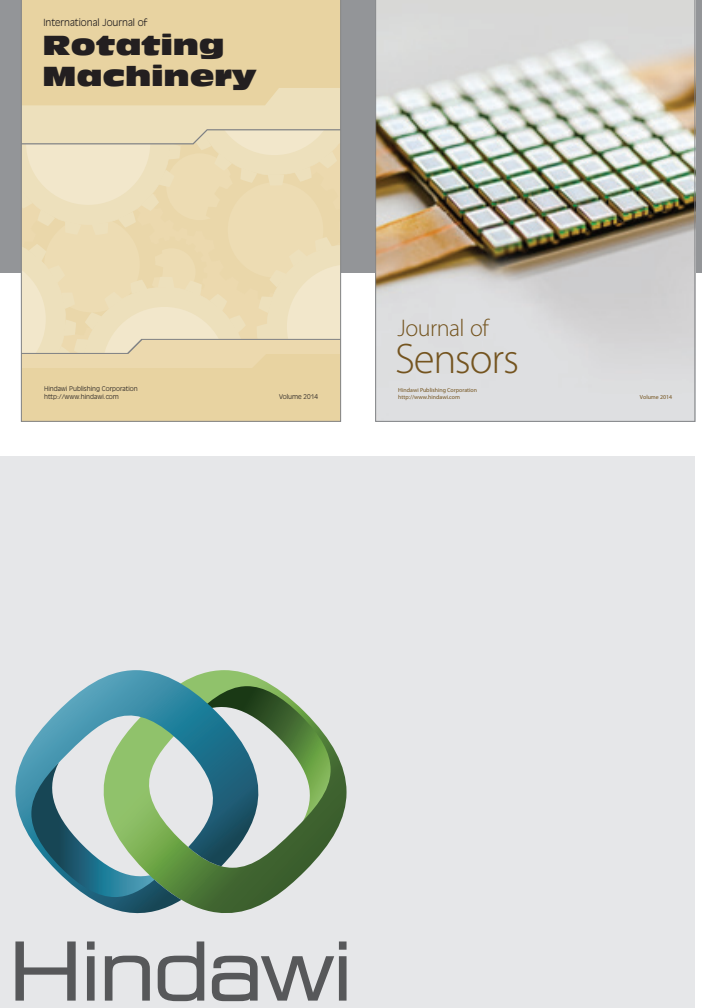

Submit your manuscripts at http://www.hindawi.com
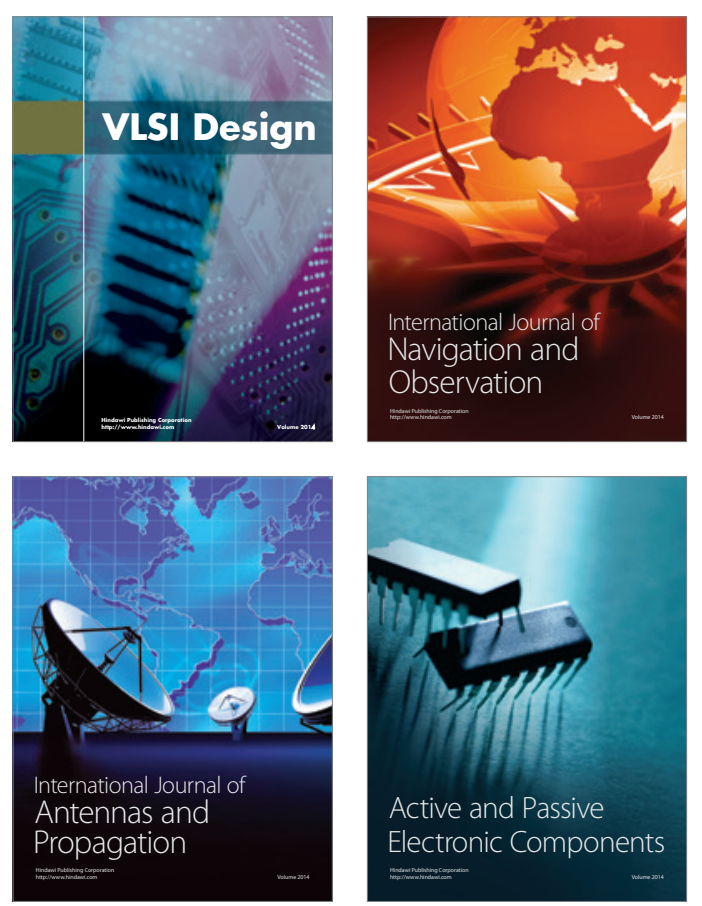
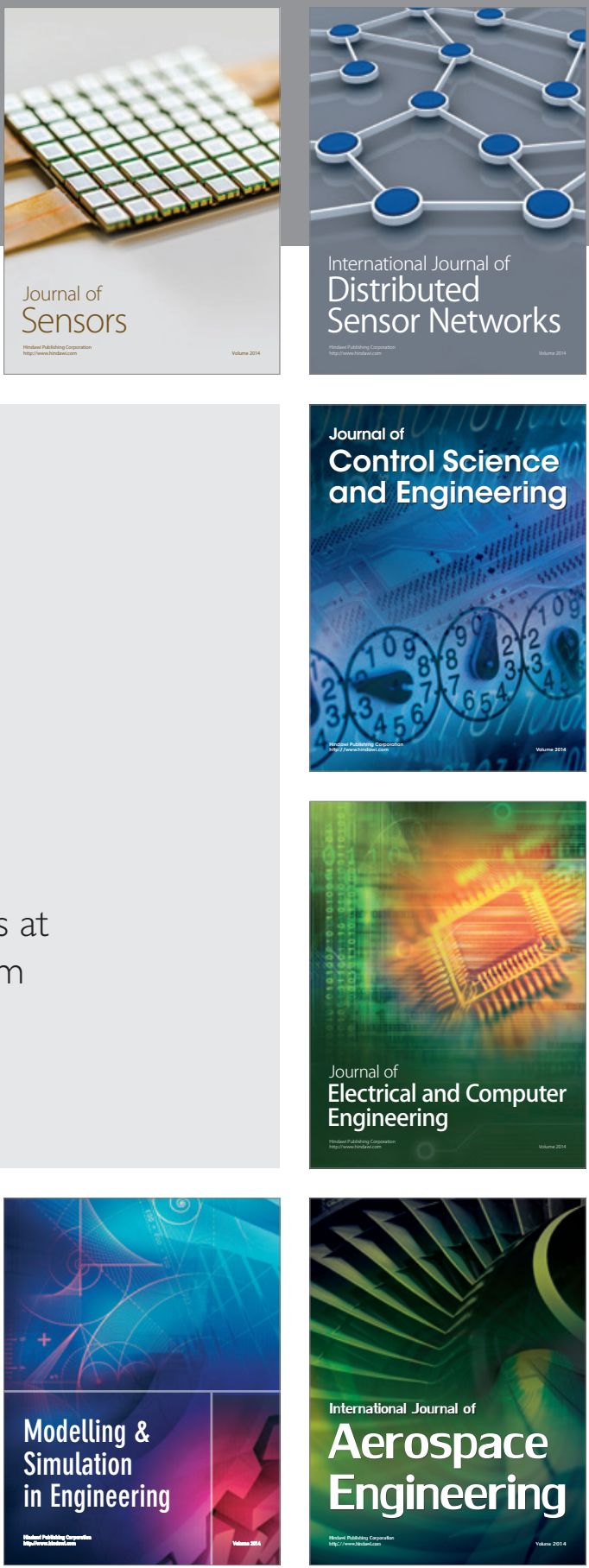

Journal of

Control Science

and Engineering
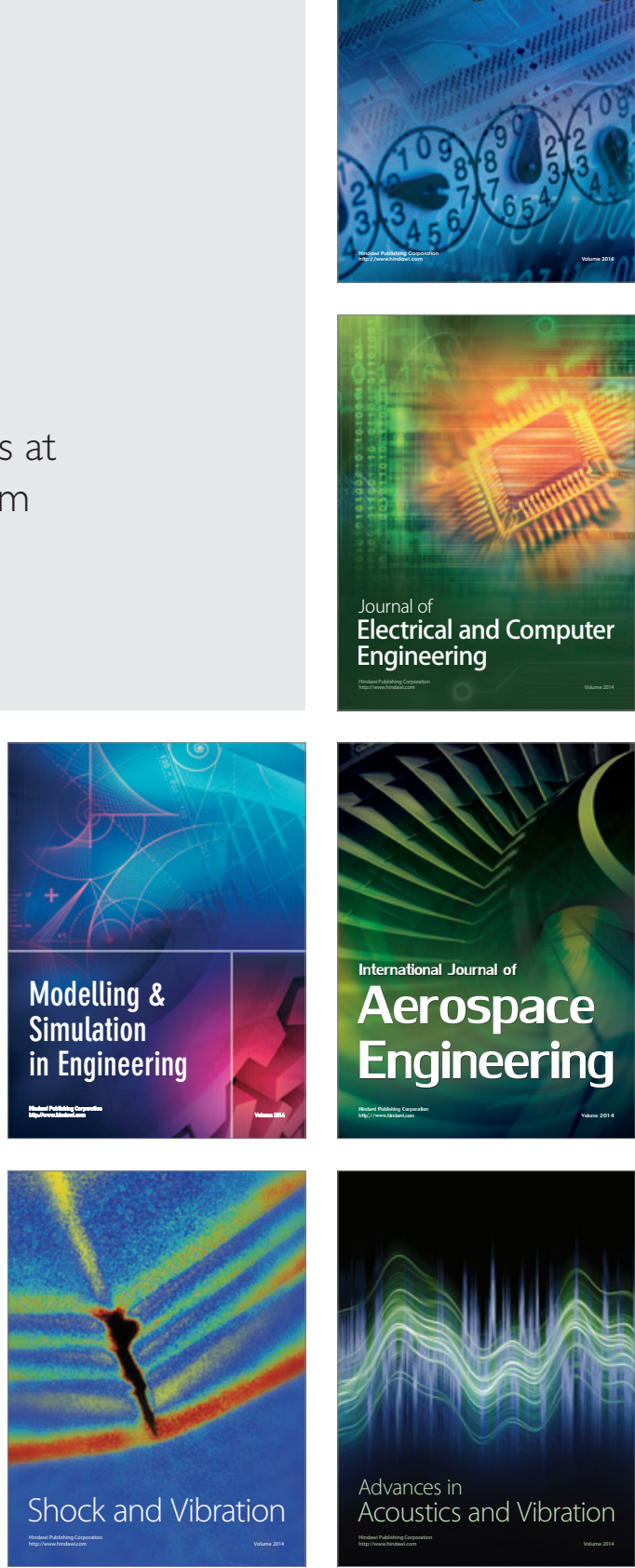